\title{
Dietary bioaccumulation of persistent organic pollutants in the common sole Solea solea in the context of global change. Part 1: Revisiting parameterisation and calibration of a DEB model to consider inter-individual variability in experimental and natural conditions.
}

\author{
Mounier Florence ${ }^{1,{ }^{*}}$, Pecquerie Laure ${ }^{3}$, Lobry Jérémy ${ }^{1}$, Sardi Adriana E. ${ }^{4}$, Labadie Pierre ${ }^{4}$, \\ Budzinski Hélène ${ }^{4}$, Loizeau Veronique ${ }^{2}$
}

1 INRAE, UR EABX, 50 av. de Verdun, 33612, Cestas CEDEX, France.

2 Ifremer, LBCO, Plouzané, France

3 Univ Brest, CNRS, IRD, Ifremer, LEMAR, F-29280 Plouzane, France

4 UMR 5805 EPOC, Université de Bordeaux I, F-33405 Talence, France

*Corresponding author : Florence Mounier, email address : florence.mounier@inrae.fr

\begin{abstract}
:
Studying adverse effects of chemical pressure on aquatic ecosystems needs a comprehensive knowledge of bioaccumulation mechanisms of pollutants in biota to predict internal concentrations, especially for Persistent Organic Pollutants (POPs). However, the large variability of responses in measured POP concentrations requires explicit consideration of both individual variability and environmental influences. Dynamic Energy Budget (DEB) theory provides a rigorous and generic conceptual framework for tackling these questions in a relevant mechanistic way. In the present study, parameterisation and calibration of previous DEB models for Solea solea were revisited in order to accurately represent the full life cycle with an original emphasis on larval stage, metamorphosis, reproduction rules and sexual differences. We first improved calibration thanks to the use of the estimation procedure developed by the DEB network coupled with a broad compilation of data from literature. Then, we validated this set of parameter estimates on independent datasets of i) individual monitoring of larval growth in controlled food conditions from a novel experiment, and ii) juvenile and adult growth, and female fecundity, from a natural population. Finally, we combined the DEB model developed in the present paper with we used a simple toxicokinetic (TK) model from literature. This TK model was also combined to a previous DEB model and was used to reproduce the mean trajectories of a growth and contamination dataset. We applied the same TK model with our DEB model considering inter-individual variability in food availability. This application highlighted the need to accurately consider inter-individual variability in ingestion to correctly estimate growth and contamination variability. The present work is the first step in the development of a mechanistic TK model that will be used in a companion paper for investigations of juvenile sole sensitivity to warming, nursery quality and prey contamination, in highly fluctuating estuarine environments.
\end{abstract}




\section{Highlights}

- A new DEB model for Solea solea is parameterised for each sex. It is calibrated from a large variety of datasets to model the full life cycle. A model validation is done on both natural and experimental growth data. Coupling a toxicokinetic and a DEB model allows studying dietary contamination. The variability in ingestion explains contamination variability in experiments.

Keywords : DEB parameter estimation, full life cycle, Solea solea, POP bioaccumulation, dietary contamination, inter-individual variability 


\section{Introduction}

In the context of global change, evaluating and predicting responses of living organisms to several environmental disturbances is crucial in environmental risk assessment.

Aquatic ecosystems and, among them, coastal and estuarine ecosystems are particularly vulnerable to global changes, especially to climate change (Hoegh-Guldberg and Bruno, 2010), and contamination (e.g., Budzinski et al., 1997; Matthiessen and Law, 2002). Coastal and estuarine ecosystems are increasingly exploited, polluted, and their biodiversity is decreasing (IPBES, 2019). Moreover, pollution has been identified as one of the main factors affecting the ecological quality of these areas, especially by altering their nursery function (see for instance Fonseca et al., 2014; Gibson, 1994; Nunes et al., 2014; Teichert et al., 2016). The effect of pollution in these habitats is such that, in Europe and especially in France, fish-based indicators of the ecological quality of transitional waters are particularly calibrated to respond to water contamination (Courrat et al., 2009; Delpech et al., 2010; Pasquaud et al., 2013).

To study the adverse effects of chemical pressure on ecosystems and to predict internal chemical concentrations, especially for Persistent Organic Pollutants (POPs), comprehensive knowledge of bioaccumulation mechanisms of pollutants in biota is a prerequisite (van der Oost et al., 2003). Polychlorinated biphenyls (PCBs) are hazardous chemicals classified as POPs. Their physicochemical properties and fate in the environment (persistence, bioaccumulation, long-range transport and toxicity) qualify them as POPs (El-Shahawi et al., 2010). In France, this group of synthetic organic chemicals was produced extensively for a broad range of industrial applications from the 1930s to the late 1980s. Although their production is banned, levels significantly higher than the OSPAR threshold are still measured in French estuaries (ROCCH, French chemical monitoring network) for these compounds. Bioaccumulation is the result of complex dynamics of environmental, ecological, and physiological factors. Consequently, developing mechanistic tools integrating the influence of physiological and environmental factors on toxicants bioaccumulation dynamics is required to produce the most reliable predictions of organisms' response under unprecedented conditions (Mackay and Fraser, 2000). To develop this kind of tools, bioaccumulation processes need to be studied in controlled conditions. However, many of such studies (Gobas et al., 1988; Burreau et al., 1997; Fisk et al., 1998; Liu et al., 2010) concluded that the large variability of responses in measured PCB concentrations requires to explicitly consider both individual variability (e.g., length, weight and lipid content) and environmental influence (e.g., diet composition). Dynamic Energy Budget (DEB) theory (Kooijman, 2010) provides a rigorous and generic conceptual framework that integrates the inter-individual variability due to physiological and environmental factors in a relevant mechanistic way. DEB models allow predicting biological traits (e.g. food ingestion, growth) of interest in toxicokinetic (TK) models, which can be further predicted in dynamic environmental conditions (concerning particularly to food quality, food availability, and temperature) over the individual life cycle.

Flatfish benthic lifestyle and their use of coastal and estuarine zones as nursery areas during their juvenile stage expose them chronically to POPs (Gilliers et al., 2006). The common sole (Solea solea, L. 1758), a highly commercial flatfish species present throughout European coasts, is often used as a proxy for the quality of nursery grounds (Gilliers et al., 2006; Le Pape et al., 2003). Research on PCBs' bioaccumulation patterns in juvenile sole was initiated by Eichinger et al. (2010). This work was the first attempt to implement a mechanistic approach to model juvenile sole dietary contamination using data from a six-month experiment consisting in contamination-decontamination regimes. In their toxicokinetic model, the uptake flux depends on biological traits dynamics (notably food ingestion and growth), themselves predicted by a mechanistic bioenergetic model based on the Dynamic Energy Budget (DEB) theory (Kooijman, 2010).

In the present study, we developed a DEB model for Solea solea that aims to mechanistically describe the processes of food consumption and growth involved in dietary contamination experiments and in the natural environment. Combining detailed experimental data and field data can be challenging. Thus, 
the methodology used aimed at ensuring that the model was relevant for both experimental and estuarine environmental studies, and for several life stages. Also, a special attention was given to investigate sources of inter-individual variability. We focused on the variability of experimental or natural conditions, especially regarding food quality and availability.

In the first part of the present work, we focused on improving the latest DEB models for common sole (Teal, 2015. AmP Solea solea version 2015/08/28; Eichinger et al., 2010). We did so by combining: i) an update of the theory on which the model is based (e.g., maturity compartment, sex-specific parameters), ii) an upgrade of the parameterization, so it would better represent some particular life stages (i.e., larval stage and metamorphosis) and differences between male and female (i.e., reproduction rules), and iii) an upgrade of the calibration, especially to predict food ingestion more accurately. We improved the DEB parameter calibration by using the estimation procedure developed by the DEB network coupled with a broad compilation of data from the literature. Finally, we validated this set of parameters using independent datasets of individual larvae growth in controlled conditions with monitored feeding (published in this work), and juvenile and adult growth and reproduction from a natural population (Teal, 2015. AmP Solea solea version 2015/08/28).

In the second part, we combined the DEB model developed in the present paper with the TK model from Eichinger et al. (2010), and applied it to the growth and contamination datasets from the same work. In addition, we simulated multiple virtual fish with several levels of alimentation to investigate if heterogeneity of feeding accessibility between individuals could lead to both observed growth and CB153 contamination variability.

A companion paper (Mounier et al., 2020) presents how the model developed here allowed us to better understand the sensitivity of juvenile sole growth and contamination by some POPs to environmental factors affected by Global Changes in estuaries.

\section{Materials and methods}

\subsection{Model description}

\subsubsection{DEB theory applied to the life cycle of Solea solea}

Only particularities of our DEB model compared to standard or previous modelling approaches are specified here. A more complete description and a schematization of the DEB modelling framework are presented in Appendix $A 0$ and all equations for state variables and fluxes are listed in Appendices $A 1$ and A2. All parameters are described in Table 1. For a more comprehensive description of the DEB theory, a full list of equations and the notation rules used, please refer to the third edition of DEB theory (Kooijman, 2010).

Metabolic acceleration. For this study, we used the typified DEB model named 'abj' recommended for species including a metamorphosis in their early life cycle. This model has already been used for several Pleuronectiformes species (Kooijman, 2014) including the common sole (Teal, 2015. AmP Solea solea version 2015/08/28, bio.vu.nl/thb/deb/deblab/add_my_pet/). This extended standard ('std') DEB model considers a type $\mathrm{M}$ metabolic acceleration of assimilation and mobilization between birth $(b)$ and metamorphosis ( $j$ ) stages (Kooijman, 2014; Lika et al., 2014). Induced changes in DEB parameters are described in Appendix A3.

Life stages. To account for the whole life cycle of common sole, five life stages were considered in our model. They were triggered when $E_{H}$ exceeded fixed threshold values corresponding to four parameters: hatching parameter $E_{H}^{h}$ (end of egg stage), birth parameter $E_{H}^{b}$ (start of feeding and metabolic acceleration), metamorphosis parameter $E_{H}^{j}$ (end of metabolic acceleration and 
metamorphosis) and puberty parameter $E_{H}^{p}$ (end of maturation and start of allocation to reproduction buffer).

Table 1 - Parameters of the DEB model with symbol, description, unit, and source of parameter estimation. Dots indicate quantities expressed per unit of time (rates), brackets per unit of structural volume and braces per unit of structural surface area. Indices $\boldsymbol{d}$ and $\boldsymbol{w}$ indicate respectively dry and wet units of mass. Generalised animal refers to a theoretical reference for maximum structural length of $1 \mathrm{~cm}$ at $\boldsymbol{T}_{\text {ref }}$. AmP calibration refers to this study (see 2.2.1).

\begin{tabular}{|c|c|c|c|}
\hline Symbol & Description & Unit & Source \\
\hline \multicolumn{4}{|c|}{ DEB core parameters } \\
\hline$\left\{\dot{p}_{A m}\right\}$ & Maximum food assimilation rate & $J \cdot \mathrm{cm}^{-2} \cdot d^{-1}$ & Derived from $\left(z\left[\dot{p}_{M}\right]\right) / \kappa$ \\
\hline$\kappa_{X}$ & Digestion efficiency of food to reserves & - & Fixed to generalized animal value \\
\hline$\dot{v}$ & Energy conductance & $c m \cdot d^{-1}$ & AmP calibration \\
\hline$\kappa$ & $\begin{array}{l}\text { Fraction of catabolic energy allocated to somatic } \\
\text { maintenance plus growth }\end{array}$ & - & AmP calibration \\
\hline$\left[\dot{p}_{M}\right]$ & Somatic maintenance rate & $J \cdot \mathrm{cm}^{-3} \cdot \mathrm{d}^{-1}$ & AmP calibration \\
\hline$\left[E_{G}\right]$ & Costs of structure & J. $\mathrm{cm}^{-3}$ & AmP calibration \\
\hline$\dot{k}_{J}$ & Maturity maintenance rate coefficient & $d^{-1}$ & Fixed to generalized animal value \\
\hline$E_{H}^{h}$ & Maturation threshold at hatching & $J$ & AmP calibration \\
\hline$E_{H}^{b}$ & Maturation threshold at birth (first feeding) & $J$ & AmP calibration \\
\hline$E_{H}^{j}$ & Maturation threshold at metamorphosis & $J$ & AmP calibration \\
\hline$E_{H}^{p}$ & Maturation threshold at puberty & $J$ & AmP calibration \\
\hline$\kappa_{\mathrm{R}}$ & Fraction allocated to gametes & - & $\begin{array}{l}\text { Fixed to generalized animal value for female } \\
\text { calibrated for male }\end{array}$ \\
\hline \multicolumn{4}{|c|}{ Temperature parameters } \\
\hline$T_{L}$ & Lower boundary of tolerance range & $K$ & Fixed (Freitas et al., 2010) \\
\hline$T_{H}$ & Upper boundary of tolerance range & $K$ & Fixed (Freitas et al., 2010) \\
\hline$T_{A}$ & Arrhenius temperature & $K$ & AmP calibration \\
\hline$T_{A L}$ & Rate of decrease at lower boundary & K & Fixed (Teal et al., 2012) \\
\hline$T_{A H}$ & Rate of decrease at upper boundary & $K$ & Fixed (Teal et al., 2012) \\
\hline \multicolumn{4}{|c|}{ Auxiliary parameters } \\
\hline$\delta_{M}^{l}$ & Shape coefficient before maturity (larvae) & - & AmP calibration \\
\hline$\delta_{M}^{a}$ & Shape coefficient after maturity (juvenile and adult) & - & AmP calibration \\
\hline$d_{V}$ & Density of structure & $g_{d} \cdot \mathrm{cm}^{-3}$ & $\begin{array}{l}\text { Fixed to default value for Actinopterygii from } \\
\text { DEBtool package }\end{array}$ \\
\hline$\varrho_{V}$ & Energy density of structure & $J \cdot g_{d}^{-1}$ & Fixed to generalized animal value \\
\hline$\varrho_{E}$ & Energy density of reserve & $J \cdot g_{d}^{-1}$ & Fixed to generalized animal value \\
\hline$\varrho_{R}$ & Energy density of reproduction buffer & $J \cdot g_{d}^{-1}$ & $\begin{array}{l}\text { Fixed to reserve and structure values for } \\
\text { female and male respectively }\end{array}$ \\
\hline
\end{tabular}

Temperature correction. In the present study, we considered the extended version of the temperature correction factor (Kooijman 2010) to allow considering conditions outside of the optimal temperature range of Atlantic population of the common sole. The five parameters $\left(T_{A}, T_{A L}, T_{A H}, T_{L}\right.$ and $\left.T_{H}\right)$ are described in Table 1 and equations are described in Appendix A4.

\subsubsection{Sexual dimorphism}

Sex differences in growth and puberty were observed for common sole (Deniel, 1981). To model these differences, as in the model for the Atlantic halibut from AmP collection (Wiedmann et al., 2017. AmP 
Hippoglossus hippoglossus version 2017/03/06, we considered different values between male and female for two parameters: (1) the maximum specific assimilation $\left\{\dot{p}_{A m}\right\}$ value to reproduce growth dimorphism, and (2) the level of maturity at puberty $\left(E_{H}^{p}\right)$. Sex difference on $\left\{\dot{p}_{A m}\right\}$ was applied throughout the life cycle. These assumptions are discussed in section 4.1.

Some additional differences between sexes were added to predict realistic weights of reproduction buffer and thus realistic total weights for adults. The auxiliary parameter of reproduction buffer energy density $\left(\varrho_{R}\right)$ was differentiated as eggs have a greater lipid content than semen in fish (Vassallo-Agius et al., 2001), leading to a higher energy density. We assumed that sexes also differ from their reproduction overheads $\left(\kappa_{R}\right)$ as suggested by Freitas et al. (2010).

\subsubsection{Reproduction rules}

Reproduction rules permit to model how and when mature fish are emitting their gametes, which is an important process to consider for future work on population dynamics and decontamination through gametes emission (Bodiguel et al., 2009; Daouk et al., 2011; Munschy et al., 2017), but outside the scope of the present study.

For mature fish $\left(E_{H}=E_{H}^{p}\right)$, energy allocated to reproduction $\left(\dot{p}_{R}\right)$ is instantly used to gamete production, stored in the reproduction buffer $\left(E_{R}\right)$ after deduction of the reproduction overheads ( $(1-$ $\left.\left.\kappa_{R}\right) \dot{p}_{R}\right)$. Gametes are stored until spawning season. According to the histological sections of gonads made by Deniel (1981), the common sole oocyte maturation cycle lasts 1 year. Thus, in the model, females reaching puberty $\left(E_{H}=E_{H}^{p}\right)$ less than 1 year before the following spawning season were not allowed to emit gametes during this first season. Indeed, Ramsay and Witthames (1996) observed that, in the recruiting year class of sole, for a large number of fish, ovaries development started but was aborted before complete maturation, probably leading to the atretic resorption of the vitellogenic oocytes. We thus assumed that this energy allocated to reproduction was entirely lost through the energetic processes of atresia. Consequently, $E_{R}$ is emptied for each sex at the end of spawning period, whether by atresia or gametes emission.

Gametes emission was triggered at given dates in the model. For female, spawning was divided into two batches of eggs to reproduce the two groups of oocytes maturing sequentially, as described by Deniel (1981). Gametes emission was triggered at dates matching with the observations by this author on spawning peaks in the Bay of Biscay: on February $1^{\text {st }}$ half of the $E_{R}$ content was emitted and on March $1^{\text {st }} E_{R}$ was emptied. Indeed, in fully matured females, atresia only concerns a small number of oocytes (Deniel, 1981). To our knowledge there is no information about the frequency of male gametes emission in the natural environment. Nonetheless, we can assume that males are mating with several females during the spawning season. Moreover, unlike females, their gonado-somatic index remains low and constant during the reproduction period (Deniel, 1981), indicating that gametes can be regularly emitted. To stagger the emptying of male reproduction buffer we supposed that males emit batches of similar size regularly during all the spawning season. We arbitrarily chose to model 10 batches (i.e., approximately one every 10 days). Each one was supposed to contain $10 \%$ of the initial $E_{R}$ content (i.e., before the emission of the first batch), apart from the last batch that also contained the energy allocated to reproduction during the spawning period, to empty the buffer at the end of this season.

\subsubsection{From state variables to observations}

As DEB state variables refer to abstract quantities, auxiliary theory is needed to link these predicted variables to measurements for model calibration and application (Kooijman, 2010). In DEB theory, physical length $\left(L_{w}\right)$ is calculated using the structural volume $(V)$ and the shape coefficient $\left(\delta_{M}\right)$ following: $L_{w}=V^{1 / 3} / \delta_{M}$. In our study, for juvenile and adult stages, the shape coefficient is constant and noted $\delta_{M}^{a}$. For larval stage, this coefficient evolved from $\delta_{M}^{l}$ at hatching $\left(E_{H}=E_{H}^{h}\right)$ to $\delta_{M}^{a}$ at metamorphosis $\left(E_{H}=E_{H}^{j}\right)$ along with the maturity buffer $\left(E_{H}\right)$ following this equation: 


$$
\delta=\delta_{M}^{l}+\frac{E_{H}-E_{H}^{h}}{E_{H}^{j}-E_{H}^{h}} \times\left(\delta_{M}^{a}-\delta_{M}^{l}\right)
$$

Total dry weight of the fish $(W)$ is the sum of model compartments dry weights: $W=W_{V}+W_{E}+W_{R}$. Dry weight of structure $\left(W_{V}\right)$ is calculated as: $W_{V}=V \times d_{V}$, where $d_{V}$ is the density of dry structure. Dry weights of reserve $\left(W_{E}\right)$ and reproduction buffer $\left(W_{R}\right)$ are calculated from the energy stored in the corresponding compartments $\left(E\right.$ and $R$ ) using their respective energy densities $\left(\varrho_{E}\right.$ and $\left.\varrho_{R}\right): W_{E}=$ $E / \varrho_{E}$ and $W_{R}=R / \varrho_{R}$. The common way to convert dry mass to wet mass is to assume constant water content. To approximate these relationships for our species we used the relationship described by Fonds et al. (1989), between Fulton's condition index and fish water content. First, we calculated a Fulton's index on dry weight $\left(K=100 \times W / L_{w}{ }^{3}\right)$ to estimate the dry content $(D$ in \%) thanks to relationship from Fonds et al. (1989): $D=40.68 \times K^{0.364}$. Then, total wet weight $\left(W_{w}\right)$ was calculated from total dry weight and dry content following the formula: $W_{w}=W /(D / 100)$.

Annual fecundity is the sum of the eggs emitted for each batch, which are computed by dividing the energy emitted from the reproduction buffer for this batch by the energy content of one egg $\left(E_{0}\right)$. However, for sake of simplicity, as no data on batch were available, for model calibration and validation we used the DEBtool function to predict daily fecundity by computing the daily energy allocated to reproduction for a given length, minus the cost of egg production, divided by the energy content of one egg. Then, we obtained the annual fecundity by multiplying the daily fecundity by 365 .

\subsection{Model calibration and validation}

\subsubsection{Model calibration}

Most of the DEB parameters were calibrated simultaneously from empirical datasets from literature using the DEBtool estimation procedure presented in Marques et al. (2018).

For parameter estimation, information on the common sole was taken from published literature and raw data from Deniel's PhD work (1981), provided by Ifremer. The zero-variate data (i.e., comprising a single data point) are given in Table S2 along with their respective sources (Day et al., 1996; Deniel, 1981; Devauchelle et al., 1987; Dorel, 1986; Fonds, 1979; Galois et al., 1990). The data includes information on egg weight and energy content, age, length and weight at hatch, birth, metamorphosis, and puberty for each sex for the last stage; ultimate length and weight for each sex; life span for each sex and maximum reproduction rate per day for female. The univariate data, comprising two dimensions observations, are given in Table S3 along with their respective sources (Day et al., 1996; Deniel, 1981; Fonds and Saksena, 1977; Lund et al., 2008) and consisted in: time series of growth in length and weight for larvae in controlled conditions; in situ time series of growth in length and length-weight relationship for each sex (Brittany, France), in situ annual egg production at different lengths (Brittany, France) and juvenile ingestion rate at different lengths and temperatures in controlled conditions.

Thus, the data used came from multiple sources that considered different living environments (aquaculture, experimentation, in situ), life stages and populations. Therefore, they were considered differently in the estimation procedure according to the purpose of our model application. Our choices about data weights used in the mydata Matlab ${ }^{\circledR}$ file (see electronic supplementary) and the relative importance in model adjustment to the different datasets are further discussed in the Supplementary Material (SM) file, section 1.1.3.

Due to the lack of detailed information in the literature, some parameters from Table 1 could not be estimated. First, some of them (e.g., digestion efficiency of food, maturity maintenance rate coefficient, reserve and structure energy densities, see Table 1) were set to typical values established for a wide variety of animals (i.e., the generalized animal values introduced by Lika et al., 2011). For the energy densities of reproduction buffer $\left(\varrho_{R}\right)$ for males and females, we assumed that egg and semen 
compositions were similar to reserve and structure compartments compositions, respectively. For female $\kappa_{R}$, a generalized animal value exists, but not for male. Thus, after the estimation of all other parameters, we chose to calibrate $\kappa_{\mathrm{R}}$ for males to reproduce the observed difference in weight loss during the reproduction period between females and males (see SM section 1.2 for detailed method). Most temperature correction parameters were fixed to literature values for our species (references used are indicated in Table 1, and assumptions made can be found in SM section 1.3). The remaining parameters were calibrated using the estimation procedure presented in Marques et al. (2018b). It consists of estimating all DEB parameters simultaneously from several empirical datasets by minimizing the weighted sum of squares deviation between model predictions and observations. We used the codes for parameter estimation developed in the DEBtool package (freely downloadable at http://www.bio.vu.nl/thb/deb/deblab/) with Matlab ${ }^{\circledR}$ program version R2016b. For this estimation, we retrieved a large set of natural and experimental data from the literature to compare with model predictions.

Matlab ${ }^{\circledR}$ files provided in supplementary materials contain all codes and data used. In combination with the DEBtool package, these files allow repeatability of the computations. The relative error (RE) of each dataset was computed and used to quantify the overall goodness of fit of the model with the Mean Relative Error (MRE in [0, $\infty$ [) and Symmetric Mean Squared Error (SMSE in [0, 1]) (Marques et al., 2018). Values of MRE and SMSE close to 0 mean that the model predictions are globally close to the data.

The estimated parameter set was then compared with those from previous DEB models for $S$. solea (Teal, 2015. AmP Solea solea version 2015/08/28; Eichinger et al., 2010; Freitas et al., 2010; van der Veer et al., 2001). Following the DEB model classification from Freitas et al. (2010), DEB type of model were labelled "DEB2" for the second version of DEB theory (Kooijman, 2000) and "DEB3" for the third one (Kooijman, 2010). Classically, DEB2 models estimated mechanistic parameters independently directly from observations of each mechanism. However, parameters that are not directly observable (e.g., energy conductance $\dot{v}$ ) couldn't be estimated properly. To estimate these parameters, DEB3 models benefitted from the covariation method (Lika et al., 2011; Marques et al., 2018a) and DEB parameters are estimated conjointly from all datasets together. The overall goodness of fit (i.e., MRE and SMSE) was also compared with the one from AmP collection as it was calibrated using the same procedure.

\subsubsection{Model validation}

\section{Larval phase}

We confronted the present DEB model to independent datasets on larval growth (from birth to metamorphosis) in controlled conditions of temperature and salinity and with individually monitored feeding. This recent experiment was carried out by A.E. Sardi as part of her Post-doctorate project. Detailed experimental design and monitoring protocols can be found in SM (section 2).

S. solea larvae were reared in microplates with constant temperature $\left(16 \pm 1{ }^{\circ} \mathrm{C}\right)$ for 33 days (posthatching). From 5 dph onwards (i.e. mouth opening), larvae were fed with Artemia salina. The number of artemias ate per larva was monitored daily. The objective was to provide food ad libitum without compromising the quality of the water. The length of 12 larvae was monitored every week, starting at 7 $\mathrm{dph}$ until the end of the experiment at $33 \mathrm{dph}$. At the end of the experiment, dry weight was measured for all larvae. A score of metamorphosis completion ranging from 0 (unstarted metamorphosis) to 9 (complete metamorphosis) was also defined for each larva depending on morphologic observations (see the methods in SM section 2.2). Raw datasets of individual daily food consumption, daily portion of food distributed having been ingested, growth monitoring, and final dry weight can be found in SM in Tables S7, S8, S9, and S10, respectively. 
We used information on the number of prey (artemias) ingested daily by each fish in order to use the DEB ingestion flux $\left(\dot{p}_{X}\right)$ as a model input directly. This flux being in energy per day, we had to transform the number of artemias ingested per day into joules ingested per day. The weight and energy density of artemias from this experiment were not measured, and the literature reported a large variability for both of them. Indeed, individual dry weight of an Artemia Nauplius (instar I) ranged from 1.63 to 3.33 $\mu \mathrm{g}$ and dry energy density from 21760 to $22480 \mathrm{J.g}{ }^{-1}$ (Vanhaecke et al., 1983). To cope with this variability, we performed two simulations for each larva using either the minimum or maximum individual energy content for a Nauplius. These values were estimated, respectively multiplying either the minimum values of weight and energy density (low food condition) or their maximums (high food condition). The validation of the model for the larval phase was performed by comparing the data on individual growth trajectories and final dry weight, to the predictions of the model for each larvae using their respective food intakes (in number of artemias per day) and either a low or high food condition. Also, two virtual fish were modelled for an ad libitum scenario using $f=1$ and either the minimum or maximum energy density of Nauplii from Vanhaecke et al. (1983).

The initiation of the simulations corresponds to larvae of $4 \mathrm{dph}$ and the day just before the first feeding in the experiment (i.e., birth stage in DEB model). To estimate the initial values for DEB state variables, we computed the DEB model at $16^{\circ} \mathrm{C}$ and $f=1$ (ad libitum) and took their values for the day just before birth stage was reached.

Simulations of growth trajectories in length and weight for each food condition were graphically compared to measurements. Predicted age at metamorphosis for each simulation and differences between individuals were compared to the observed scores of metamorphosis completion.

\section{Juvenile and adult phases}

We compared the present DEB model to independent datasets on juvenile and adult growth, and female fecundity, from a natural population that was different from the one used for calibration. Data on agelength and length-fecundity relationships were obtained from the univariate-data used to calibrate the model for Solea solea from the AmP collection (Teal, 2015. AmP Solea solea, version 2015/08/28). The original source of the age-length relationship was estimated from an IMARES (Institute for Marine Resources \& Ecosystem Studies) database called "Frisbe" containing otolith back-calculation data for females. Teal (2015) explored this database in 2011 and estimated an age-length relationship. The original source of the length-fecundity relationship is Witthames et al. (1995). They estimated potential annual fecundity from histological method applied to the ovaries of females sampled in the ICES division IVb East in 1991. To compare model predictions to these datasets, Teal assumed that the mean annual temperature was $10{ }^{\circ} \mathrm{C}$ for the North sea and estimated a different value of scaled functional response $(f)$ for each dataset.

For the purpose of model validation, predictions were obtained using the DEB estimates set calibrated in this work, apart from the environmental parameter characterizing food availability $(f)$. This parameter was estimated for each one of the two datasets using the DEBtool package. The temperature used was the same one used in the 2015-AmP model for these data. We also compared model predictions with an additional fecundity dataset from an adjacent area (IVb West), from the same source (Witthames et al., 1995).

\subsection{Model application}

For model application on juvenile phase, we used data from Eichinger et al. (2010) on growth performance and internal concentration in CB153 and CB105 during a dietary exposure experiment of common sole juveniles to a mixture of PCBs. A summary of the material and methods information necessary for model application from this contamination-decontamination experiment is presented in SM (section 3). 
We investigated if heterogeneity of feeding accessibility between individuals could lead to both observed growth and contamination variability. For that purpose, we applied the present DEB model for several virtual fish characterized by different food levels and different initial weights. We compared growth predictions with the observed variability in weight between individuals monitored from the onset to the end of Eichinger et al. (2010) experiment. To investigate the influence that these two types of variability sources exert on bioaccumulation, we applied the bioaccumulation model from Eichinger et al. (2010) using the present DEB model. PCB concentration in fish $[C B]_{\text {fish }}^{\#}$ was computed for each CB (\#) following these formulas:

$$
\frac{d C B_{\text {fish }}^{\#}}{d t}=\frac{[C B]_{\text {food }}^{\#}}{\mu_{\text {food }}} \dot{p}_{A}
$$

and

$$
[C B]_{f i s h}^{\#}=\frac{C B_{f i s h}^{\#}}{W_{w}}
$$

With $C B_{\text {fish }}^{\#}$ the fish body burden (ng), $[C B]_{\text {food }}^{\#}$ the concentration in food (ng.gfood $\left.{ }^{-1} w w\right), \mu_{\text {food }}$ the food energy density (J.gfood ${ }^{-1} \mathrm{Ww}$ ), $\dot{p}_{A}$ the assimilation flux of food (DEB model prediction) and $W_{w}$ the fish total wet weight (g ww). This toxicokinetic model implies that PCBs are (1) as assimilated as food (i.e., $80 \%$ of the ingestion), and (2) not eliminated but can only be diluted by growth.

The range of weight at the onset of the experiment was defined using the $95 \%$ confidence interval of the population of dosed fish at the onset. Thus, we assumed that this sample was representative of a normally distributed population of fish whose median weight is equal to $16.0 \mathrm{~g}$ and standard deviation equal to $4.6 \mathrm{~g}$. We computed simulations for two initial weights corresponding to the mean weight of this population \pm 1.96 times the standard deviation.

The range of variations of food availability for the different fish in tank was simulated by the use of extremum $f$ values (i.e., the smallest value not leading to growth costs that would be larger than the somatic part of the catabolization flux and the ad libitum value assumed to be equal to 1 ), for each virtual fish. This value was kept constant during the experiment.

Model predictions for the six combinations of initial weights and food conditions were graphically confronted to individual measurements of total wet weight and to pooled individuals measurements of CB153 and CB105 contamination levels.

\subsection{Implementation}

Model calibration and validation on juvenile and adult phases were performed using software package DEBtool (in Matlab ${ }^{\circledast}$ ). Simulations for model validation on larval phase and model application were implemented using $R$ language ( $R$ core team, 2017) version 3.6.1 and the integrated development environment (IDE) R-studio version 1.2.5019.

\section{Results}

\subsection{Model calibration}

The parameter estimates of the present DEB model are presented along with those from previous DEB models for $S$. solea in Table 2. Compared to previous estimation sets, our estimation procedure led to an intermediate set between DEB2 and DEB3 models. Indeed, DEB2 sets were characterized by higher $\left\{\dot{p}_{A m}\right\}, \dot{v},\left[\dot{p}_{M}\right]$ and $\left[E_{G}\right]$ values than those from DEB3 models. Despite those differences, $\kappa$ estimates 
from all models were close (from 0.70 to 0.77 ) apart from the estimate of van der Veer et al. (2001) (n.b., this value was approximated but not estimated for $S$. solea by the authors). Newly estimated parameter of male fraction of energy allocated to gametes $\left(\kappa_{R}\right)$ was more than twice lower than female one.

The parameter estimation resulted in an overall good fit between predictions and observations used for calibration (MRE $=0.187$, SMSE $=0.173$ ). Model fits to all datasets used for the calibration, the relative errors (RE) associated, and the environmental forcings used are presented in SM section 1.1.2.

Table 2 - DEB parameters estimates (optimized ones in bold) with those from previous DEB models for $S$. solea. For abj models, $\left\{\dot{\boldsymbol{p}}_{A m}\right\}$ and $\dot{\boldsymbol{v}}$ are given for metamorphosed fish (after acceleration, corrected by the acceleration factor $\left(\boldsymbol{s}_{\mathcal{M}}\right.$, see appendix A3) at metamorphosis for $f=1 ; s_{\mathcal{M}}=2.7612$ for our model) to be compared with standard (std) models estimates. Rates are given at the reference temperature $\left(\boldsymbol{T}_{\text {ref }}=20^{\circ} \mathrm{C}\right.$, literature values have been corrected when necessary).

\begin{tabular}{|c|c|c|c|c|c|c|}
\hline \multirow[t]{2}{*}{ Symbol } & \multicolumn{2}{|c|}{$\begin{array}{l}\text { This study } \\
\text { DEB3 - abj }\end{array}$} & \multirow[t]{2}{*}{$\begin{array}{c}\text { van der Veer al. } \\
(2001) \\
\text { DEB2 - std }\end{array}$} & \multirow[t]{2}{*}{$\begin{array}{c}\text { Eichinger et al. } \\
(2010) \\
\text { DEB2 - std }\end{array}$} & \multirow[t]{2}{*}{$\begin{array}{c}\text { Freitas et al. } \\
(2010) \\
\text { DEB3 - std }\end{array}$} & \multirow[t]{2}{*}{$\begin{array}{c}\text { AmP Solea solec } \\
\text { version (2015) } \\
\text { DEB3 - abj }\end{array}$} \\
\hline & Female & Male & & & & \\
\hline \multicolumn{7}{|c|}{ DEB core parameters } \\
\hline$\left\{\dot{\boldsymbol{p}}_{A m}\right\}$ & 710 & 671 & 687 & 652 & 200 & 332 \\
\hline$\kappa_{X}$ & \multicolumn{2}{|c|}{0.8} & 0.8 & 0.8 & 0.8 & 0.8 \\
\hline$\dot{v}$ & \multicolumn{2}{|c|}{0.0724} & 0.2748 & 0.2247 & 0.0196 & 0.0112 \\
\hline $\boldsymbol{\kappa}$ & \multicolumn{2}{|c|}{0.7682} & 0.9 & 0.7 (Male: 0.64) & 0.7700 & 0.7353 \\
\hline$\left[\dot{\boldsymbol{p}}_{M}\right]$ & \multicolumn{2}{|c|}{39.18} & 58.55 & 32.08 & 18.9 & 22.5 \\
\hline$\left[E_{G}\right]$ & \multicolumn{2}{|c|}{5430} & 7000 & 7000 & 5600 & 5222 \\
\hline$\dot{k}_{J}$ & \multicolumn{2}{|c|}{0.002} & $\varnothing$ & $\varnothing$ & 0.002 & 0.002 \\
\hline$E_{H}^{h}$ & \multicolumn{2}{|c|}{0.104} & $\varnothing$ & $\varnothing$ & $\varnothing$ & $\varnothing$ \\
\hline$E_{H}^{b}$ & \multicolumn{2}{|c|}{0.285} & $\varnothing$ & $\varnothing$ & 0.275 & 0.06 \\
\hline$E_{H}^{j}$ & \multicolumn{2}{|c|}{6.039} & $\varnothing$ & $\varnothing$ & $\varnothing$ & 3.92 \\
\hline$E_{H}^{p}$ & 150600 & 127206 & $\varnothing$ & $\varnothing$ & 166 & 151495 \\
\hline$\kappa_{\mathrm{R}}$ & 0.95 & 0.40 & 0.9 & 0.8 (Male: 0.2 ) & 0.95 & 0.95 \\
\hline \multicolumn{7}{|c|}{ Temperature parameters } \\
\hline$T_{L}$ & \multicolumn{2}{|c|}{276} & $\varnothing$ & $\varnothing$ & $\varnothing$ & $\varnothing$ \\
\hline$T_{H}$ & \multicolumn{2}{|c|}{303} & $\varnothing$ & $\varnothing$ & $\varnothing$ & $\varnothing$ \\
\hline$T_{A}$ & \multicolumn{2}{|c|}{5119} & 8500 & 4700 & 8500 & 6114 \\
\hline$T_{A L}$ & \multicolumn{2}{|c|}{50000} & $\varnothing$ & $\varnothing$ & $\varnothing$ & $\varnothing$ \\
\hline$T_{A H}$ & \multicolumn{2}{|c|}{100000} & $\varnothing$ & $\varnothing$ & $\varnothing$ & $\varnothing$ \\
\hline \multicolumn{7}{|c|}{ Auxiliary parameters } \\
\hline$\delta_{M}^{l}$ & \multicolumn{2}{|c|}{0.166} & $\varnothing$ & $\varnothing$ & $\varnothing$ & $\varnothing$ \\
\hline$\delta_{M}^{a}$ & \multicolumn{2}{|c|}{0.171} & 0.192 & 0.204 & 0.109 & 0.149 \\
\hline$d_{V}$ & \multicolumn{2}{|c|}{0.2} & 0.2 & 0.2 & 0.157 & 0.2 \\
\hline$\varrho_{V}$ & \multicolumn{2}{|c|}{20920} & $\varnothing$ & $\varnothing$ & $\varnothing$ & $\varnothing$ \\
\hline$\varrho_{E}$ & \multicolumn{2}{|c|}{23012} & $\varnothing$ & $\varnothing$ & $\varnothing$ & $\varnothing$ \\
\hline$\varrho_{R}$ & 23012 & 20920 & $\varnothing$ & $\varnothing$ & $\varnothing$ & $\varnothing$ \\
\hline
\end{tabular}

The model predicted particularly well the larval growth patterns (Figure 1a-b) with the relative errors being 0.12 and 0.08 for length and weight, respectively. The model captured well juvenile and adult growth (Figure 1c) with the predicted growth curves accurately matching the mean growth patterns of females and males ( $R E=0.07$ for both). A slightly higher deviation was observed for female reproductive output (Figure $1 \mathrm{~d}$ ) with a relative error of 0.20 . Indeed, annual egg production was overestimated for the females from 34 to $38 \mathrm{~cm}$, approximately. 

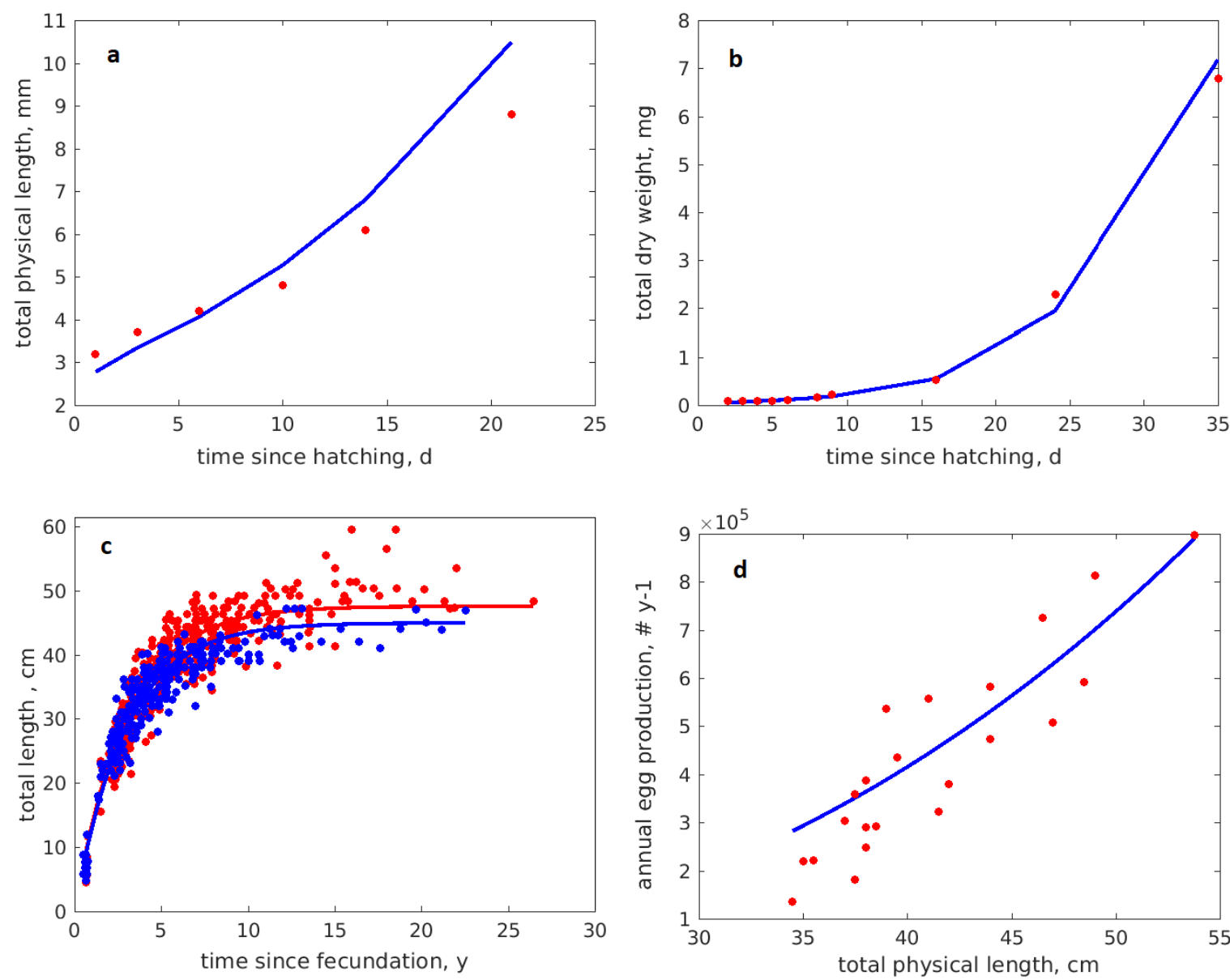

Figure 1 - Comparison of model predictions (lines) to observations (points): a. Length-at-age during larval stage at constant temperature from Lund et al. (2008). b. Weight-at-age during larval stage at constant temperature from Day et al. (1996). c. In situ length-at-age during juvenile and adult stages from Deniel (1981) with females in red and males in blue. d. In situ annual egg production as function of length from Deniel (1981).

Early developmental aspects were less well captured by the model (see Table S2). Marked deviations were especially recorded for age and weight at hatching ( $R E=0.55$ and 0.46 , respectively), age at birth $(\mathrm{RE}=0.41)$, and regarding temperature response for these life stages $(\mathrm{RE}=0.53$ for 'Tah' and $\mathrm{RE}=0.43$ for 'Tab', see univariate data from Table S3). Compared to age, length at birth and hatching were better estimated ( $R E=0.26$ and 0.21 ). Metamorphosis stage was the early life stage with the better fit for age, length, weight, and temperature response, with relative errors of $0.24,0.10,0.02$, and 0.19 , respectively.

Puberty stage was better predicted for male $(\mathrm{RE}=[0.08-0.10])$ than female $(\mathrm{RE}=[0.17-0.38])$. For female, it was the wet weight that had the worst fit $(R E=0.38)$. Also, the maximum reproduction rate was less well predicted $(\mathrm{RE}=0.4)$.

Overall, the model performed particularly well at predicting juvenile food consumption at several temperatures (RE between 0.03 and 0.17 for temperatures between 10 and $22{ }^{\circ} \mathrm{C}$, see Table S3). However, at $26{ }^{\circ} \mathrm{C}$ the model overestimated food ingestion with increasing length (see Table S3), indicating that toward the upper edge of the temperature tolerance range the model predictive capacity is probably reduced.

\subsection{Model validation}




\subsubsection{Larval phase}

In Figure 2, we compared the length observations on larval growth trajectories (linked black points) to our model simulations for each larva using either the low or high food condition (blue and orange curves, respectively). The observations were also compared to the ad libitum predictions with low and high Nauplii energy density. The simulations with low and high food condition delineate the area of possible predictions with this food condition range. Figure 3 shows similar comparisons but using the final dry weight of larvae. The low and high food conditions computed were equal to $3.510^{-2}$ and $7.510^{-2}$ J.artemia ${ }^{-1}$, respectively. For all simulations, initial values for DEB state variables were: $E=1.1 \mathrm{~J}, V=$ $1.6 \times 10^{-4} \mathrm{~cm}^{3}, E_{H}=0.258 \mathrm{~J}$, and $E_{R}=0 \mathrm{~J}$.

Simulations suggested that larvae were not fed ad libitum, which was in accordance with the observed portions of food distributed that had been ingested during the day, that were equal to $100 \%$ for more than $50 \%$ of the observations (see Table S8). The model performed well at predicting final weight interindividual variability as the different simulations with the two extreme food conditions wrapped around observations pretty well (Figure 3). Contrary to weight predictions, length predictions were less well adjusted to observed growth trajectories. First observations were underestimated for both food conditions whereas last observations were well predicted by the low food condition and overestimated by high food condition (Figure 2).

For a given food condition, predicted inter-individual variability at $33 \mathrm{dph}$ was much more underestimated for weight (Figure 3) than for length (Figure 2) (i.e., standard deviations were underestimated by a factor 4.4 and 2, for weight and length, respectively).

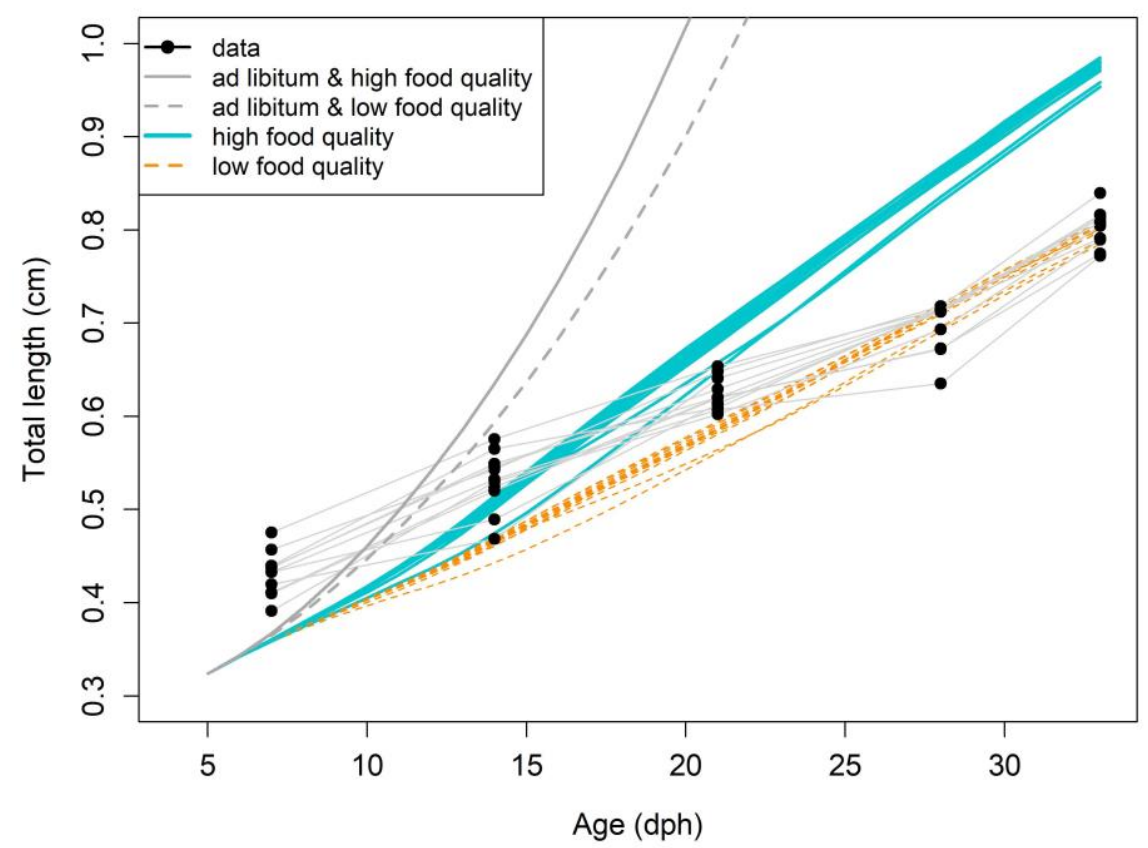

Figure 2 - Comparison of larval growth predictions in length for each larva and the two extreme assumptions for food quality conditions (blue and orange curves, see 2.2.2) to data of growth monitoring (linked points) and model prediction with ad libitum condition (grey curves). The simulations with low and high food quality delineate the area of possible predictions with this food condition range. 


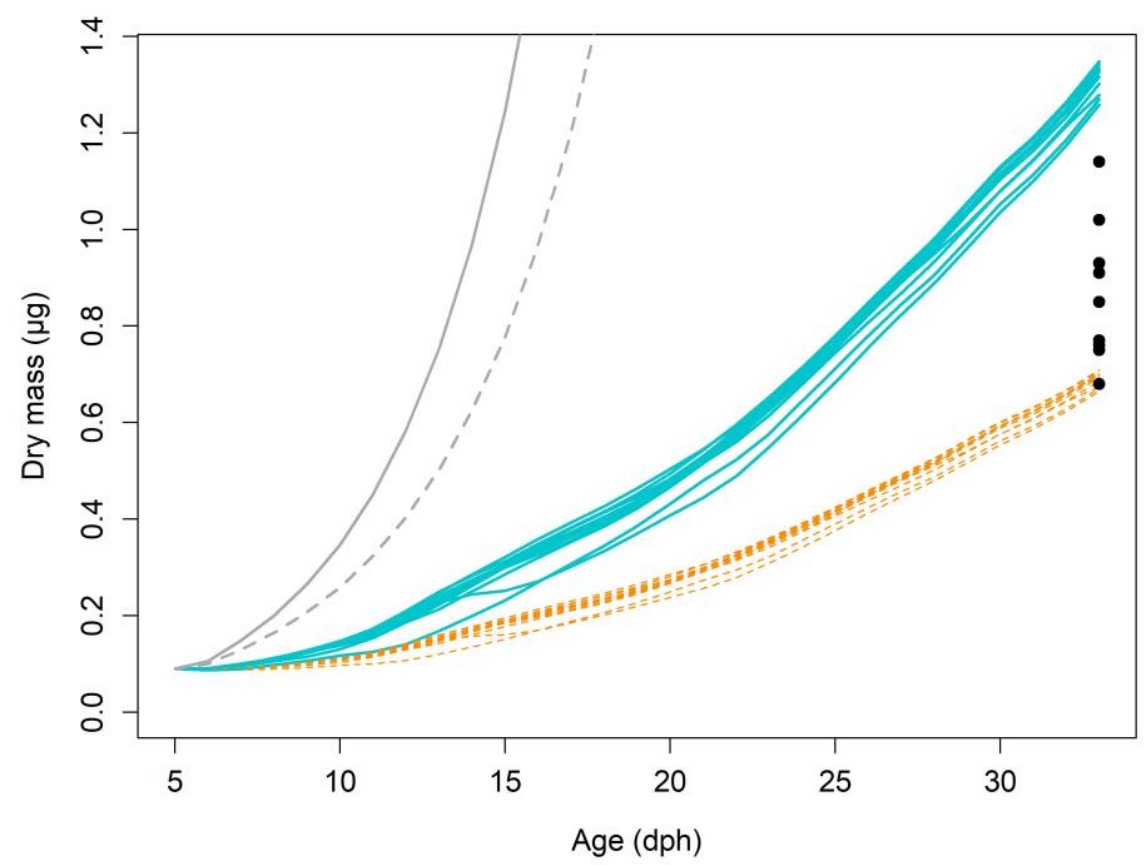

Figure 3 - Comparison of larval growth predictions in dry weight for each larva and the two extreme assumptions for food quality conditions (blue and orange curves, see 2.2.2) to data of final dry weight (black points) and model prediction with ad libitum condition (grey curves). The simulations with low and high food quality delineate the area of possible predictions with this food condition range.

Table 3 summarizes the predicted age at metamorphosis for each fish at each food scenario, together with the stage of metamorphosis achieved by day 33 post-hatching. None of the larvae simulated with low food condition reached metamorphosis, whereas all simulations with high food condition reached it. None of the larvae observed reached the maximum completion score (i.e., 9). Thus, observations were comprised between low and high food condition predictions of metamorphosis.

The relationship between predicted age at metamorphosis and observed completion score was not clear. The individual with the highest metamorphosis completion score (2_C1) matched with its simulation, that led to one of the youngest age at metamorphosis. On the contrary, the oldest predicted ages at metamorphosis (3_A1 and 2_A1) did not correspond to all the least visually developed larvae (i.e., 2_B1 was the one with the lowest score).

Table 3 - Predicted age at metamorphosis (dph) for low and high food conditions compared to the observed stage of completion of metamorphosis at $33 \mathrm{dph}$ for each of the larva considered (TAG).

\begin{tabular}{c|ccccccccccc} 
TAG & 1_A1 & 1_B1 & 1_C1 & 1_D1 & 2_A1 & 2_B1 & 2_C1 & 2_D1 & 3_A1 & 3_B1 & 3_C1 \\
\hline Low condition & NA & NA & NA & NA & NA & NA & NA & NA & NA & NA & NA \\
$\begin{array}{c}\text { High condition } \\
\begin{array}{c}\text { Metamorphosis } \\
\text { completion score }\end{array}\end{array}$ & 29.6 & 29.7 & 29.3 & 29.4 & 30.4 & 29.4 & 29.1 & 29.4 & 30.6 & 29.4 & 29.1 \\
& 5 & 5 & 5 & 6 & 4 & 3 & 7 & 5 & 4 & 5 & 5
\end{tabular}

\subsubsection{Juvenile and adult phases}

We validated the present-AmP model for the juvenile and adult phases with the datasets used for the 2015-AmP calibration ("AmP Solea solea version 2015/08/28"). We compared the present-AmP and the 
2015-AmP model adjustments. The estimation procedure of $f$ value needed to confront model predictions to datasets resulted in a value of 0.529 for the age-length dataset (Figure 4) and a value of 0.774 for the length-fecundity dataset (Figure 5).

The present model better predicted the shape of the growth trajectory compared to the 2015-AmP model (Figure 4) with the relative errors being 0.03 and 0.12 , respectively. On the contrary, model adjustment to fecundity was better for the 2015-AmP model (Figure 5) with a relative error of 0.17 (the relative error being 0.46 for the present model). The present model underestimated fecundity with increasing length. However, the 2015-AmP model did not predict that puberty could be reached for the smallest fish, whereas the present model did (i.e., puberty length of $26.3 \mathrm{~cm}$ and $23.5 \mathrm{~cm}$, respectively). With the $f$ values considered, reserve energy in one eggs $\left(E_{0}\right)$ was equal to $2.54 \mathrm{~J}$ for present-AmP and $0.34 \mathrm{~J}$ for 2015-AmP. Model underestimation was reduced when comparing predictions with fecundity data from the adjacent division of the North Sea (Figure 5).

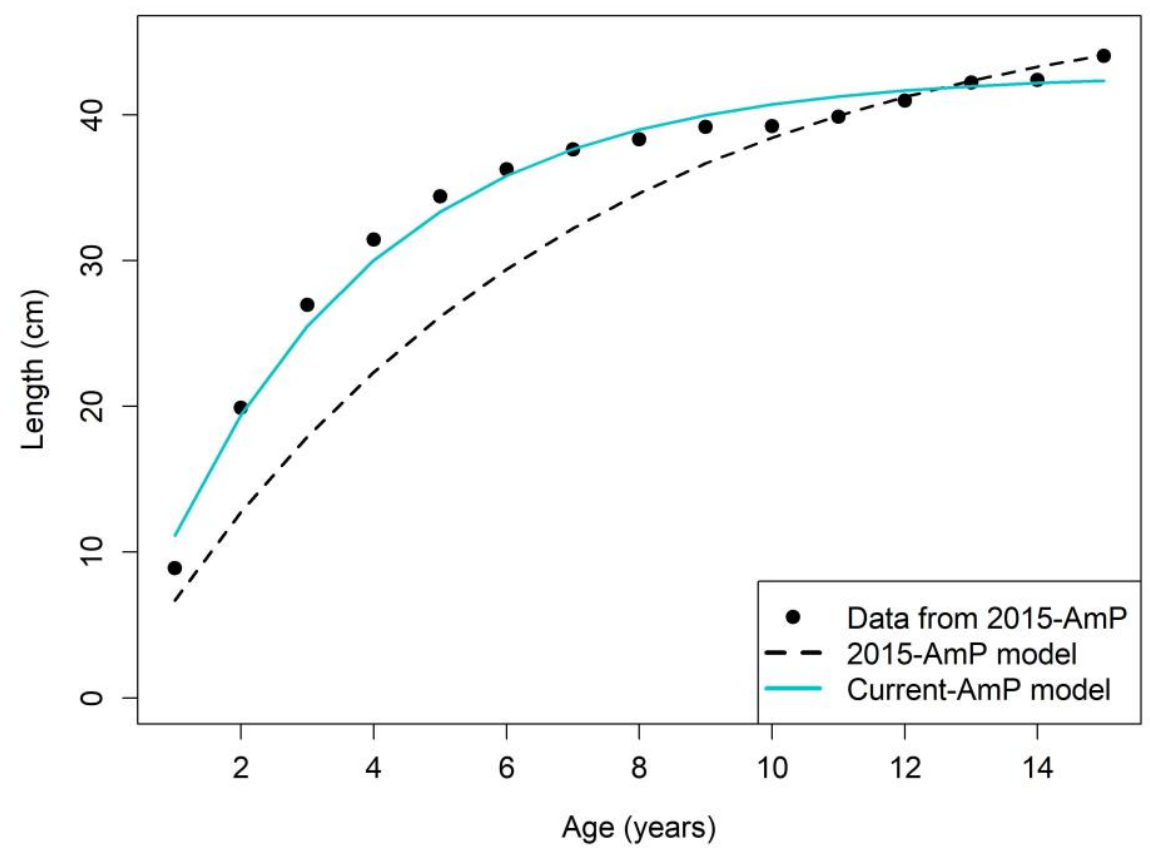

Figure 4 - Age-length relationship from AmP Solea solea dataset (black points) compared to the present model simulation (plain blue line, $\boldsymbol{f}=0.529, \mathrm{RE}=0.029$ ) and the AmP model simulation (dashed black line, $\boldsymbol{f}=0.675, \mathrm{RE}=0.1205$ ). 


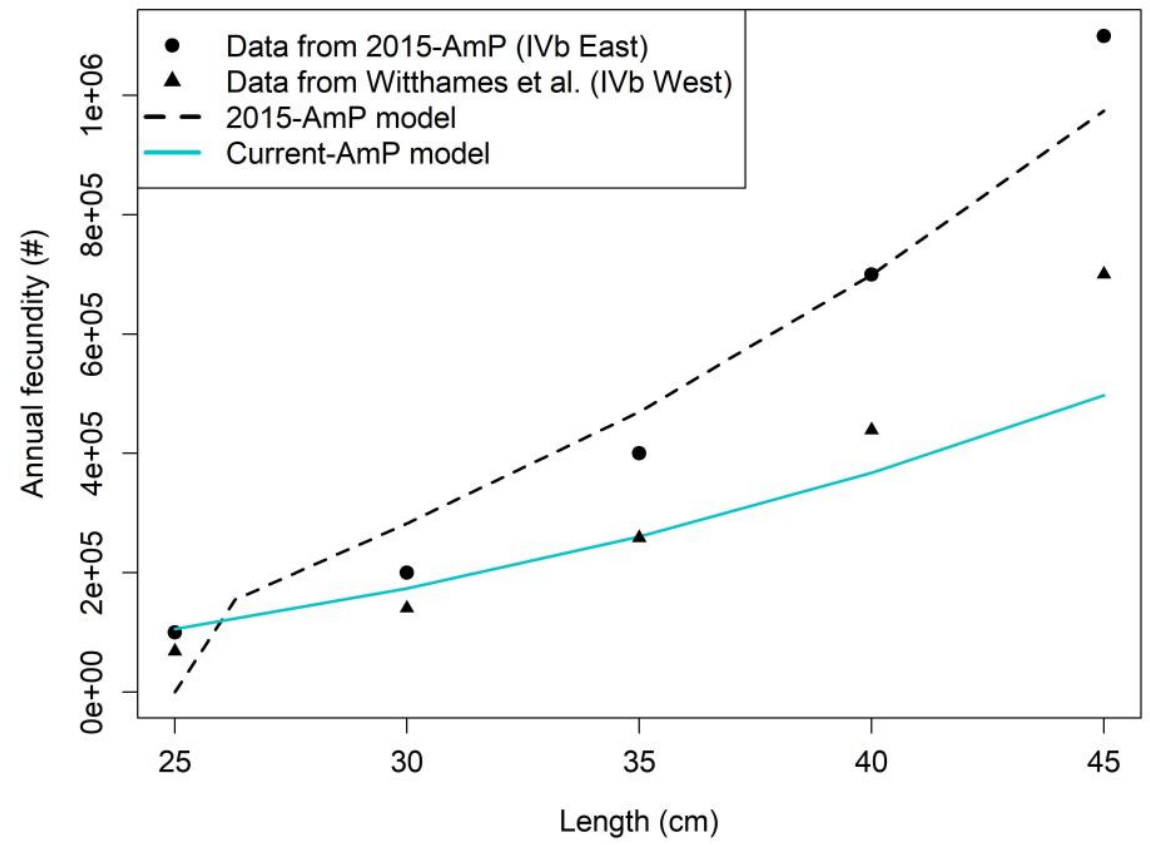

Figure 5 - Length-annual fecundity relationship from 2015-AmP Solea solea dataset (black points) compared to the present model simulation (plain blue line, $\boldsymbol{f}=0.774, \mathrm{RE}=0.457$ ) and the AmP model simulation (dashed black line, $\boldsymbol{f}=0.5286, \mathrm{RE}=0.1652$ ). Complementary data from Witthames et al. (1995) are in black triangles.

\subsection{Model application}

We compared our model growth predictions, using different food levels and initial weights, to the interindividual variability observed during the experiment of dietary exposure to PCBs from Eichinger et al. (2010). The minimum value of $f$ not leading to growth costs that would be larger than the somatic part of the mobilization flux was equal to 0.2 . The eight model simulations corresponding to the combination of the two initial weights and the two food conditions, for each sex, are plotted along with individual measurements of weight in Figure 6 . The envelope formed by the different combinations of conditions covered a large majority of the observations ( $96 \%, n=442)$. The higher the food condition, the more initial inter-individual variability was amplified over time. However, when considering only one food condition, even with the highest one, predicted variability at the end of the experiment was underestimated. 


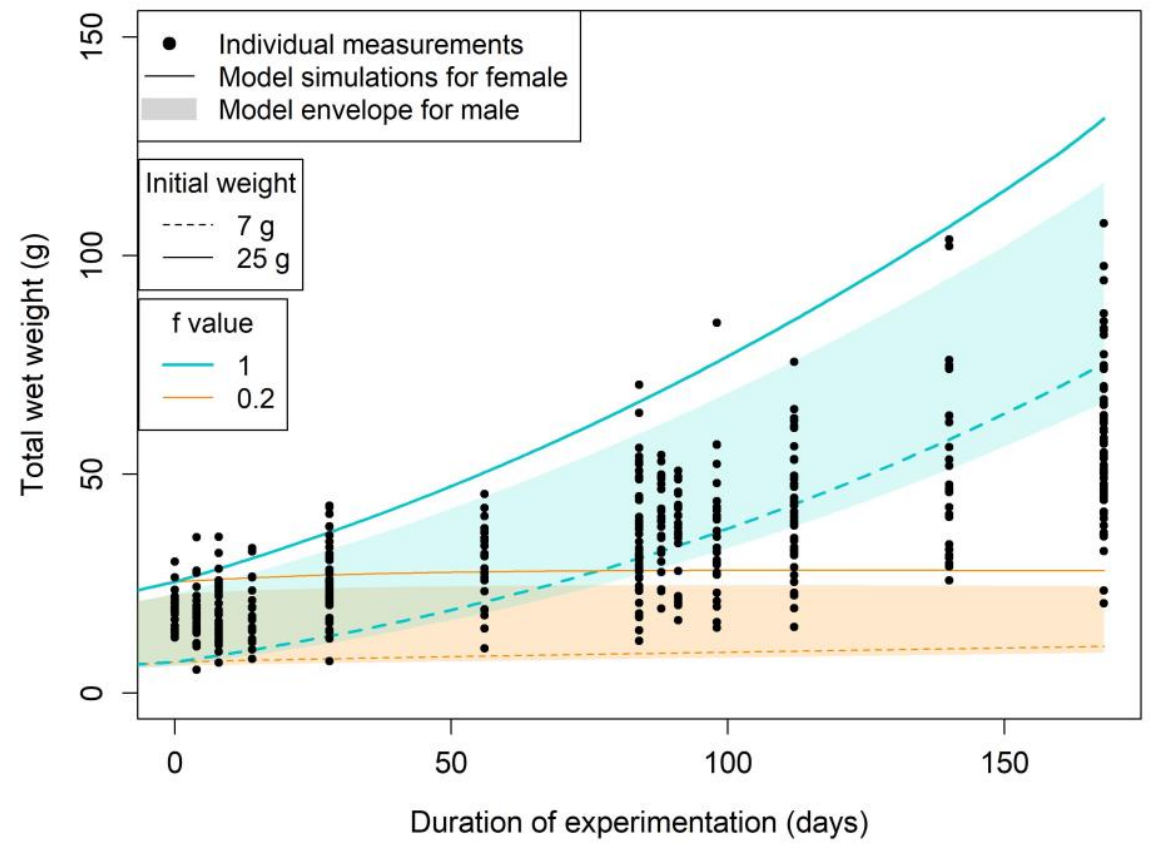

Figure 6 - Model simulations for growth with several food conditions ( $f$ value) and initial weights compared to individual biometric measurements of total wet weight ( $n=34 \pm 13$ fish for each sampling day). The lowest and the highest simulated trajectories delineate the area of possible predictions with this food availability range and this initial weight range. These trajectories are plotted as lines for females and as areas for males.

We combined the food ingestion kinetics associated to the growth simulations presented above with the TK model from Eichinger et al. (2010). We compared the resulting predictions of accumulation and depuration kinetics with measured chemical data from pooled individuals from the same experiment. Figure 7 and Figure 8 show, respectively, the predicted CB153 and CB105 contamination levels of fish during the experiment. The predicted envelopes for male and female were similar. They covered $57 \%$ of observations for CB153 and $46 \%$ for CB105 ( $n=37$ for both). Model adjustment was similar for the two contaminants. For both of them, considering the whole envelope, predicted inter-individual variability increased during the contamination period to reach a 2.8 fold difference, whereas this variability decreased to a 1.3 fold difference during the decontamination period. The same trend was observed for measurements but the variability was approximately twice the one predicted (i.e., 5.7 and 2.3 fold differences at 84 and 168 days, respectively). Also, initial inter-individual variability (for a given $f$ value) induces an increasing difference of contamination level during the contamination period, approximately in the same way for the two food conditions: the smaller the fish is at the onset of contamination the more contaminated it gets by day 84 . On the contrary, during the decontamination period, the difference between simulations decreases over time. The decontamination is quicker for high food condition and smaller fish. At the end of the decontamination period, these trends lead to (1) lower contamination levels of the smallest fish compared to the bigger ones for the high food condition and to (2) lower contamination for all the fish from high food condition compared to the bigger fish from low food condition. 


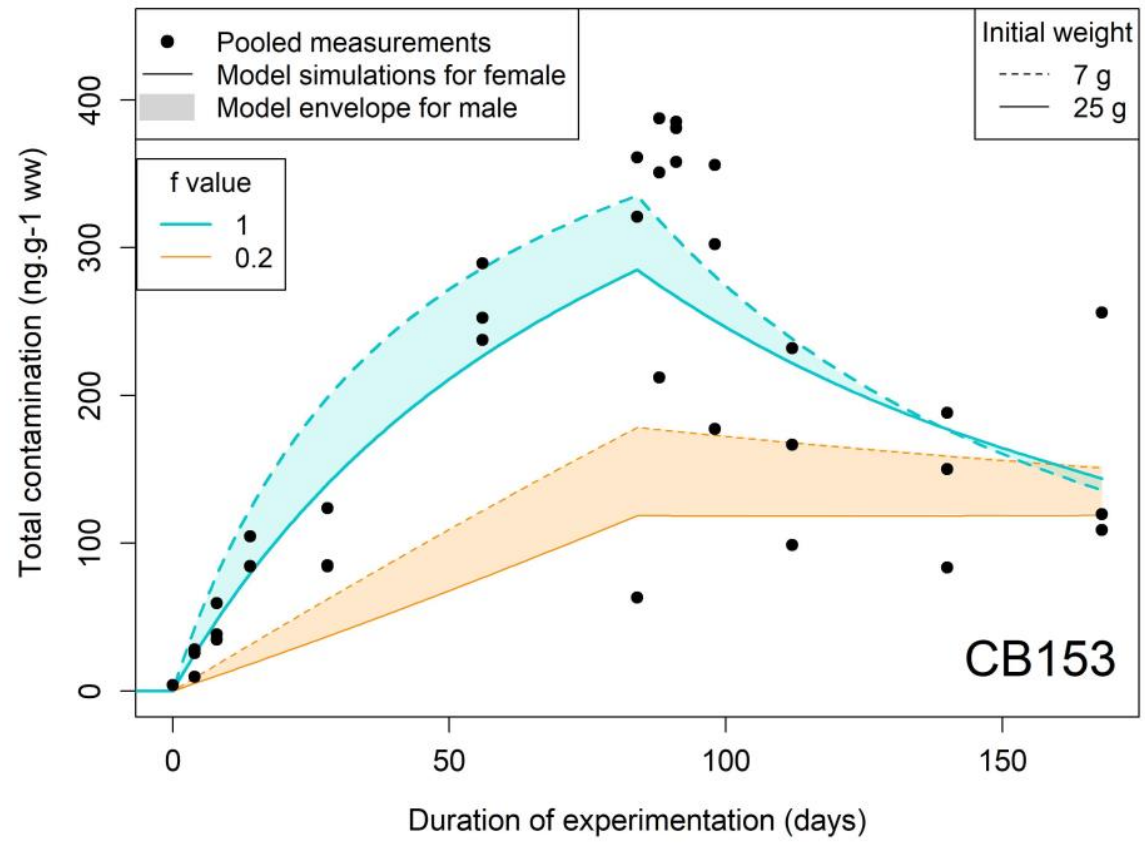

Figure 7 - Model simulations for CB153 contamination with several food conditions ( $f$ value) and initial weights compared to measurements for 3 groups (triplicates) of 4 to 11 fish on each sampling day. The lowest and the highest simulated trajectories delineate the area of possible predictions with this food availability range and this initial weight range. These trajectories are plotted as lines for females and as areas for males.

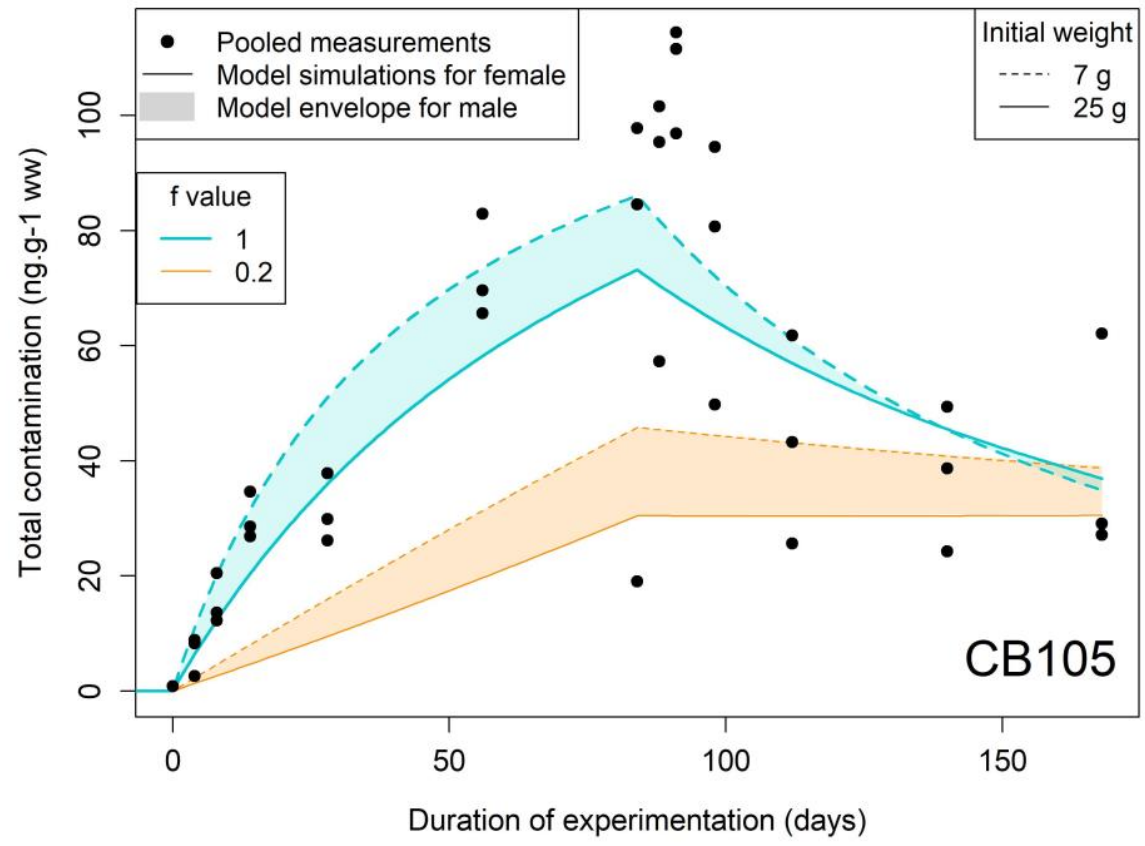

Figure 8 - Model simulations for CB105 contamination with several food conditions ( $\boldsymbol{f}$ value) and initial weights confronted to measurements for 3 groups (triplicates) of 4 to 11 fish on each sampling day. The lowest and the highest simulated trajectories delineate the area of possible predictions with this food availability range and this initial weight range. These trajectories are plotted as lines for females and as areas for males.

\section{Discussion}




\subsection{Improvements of the Solea solea life cycle model}

\section{Overall adjustment}

This study allowed us improving the parameterisation and calibration of a sole DEB model compared to the latest DEB model for S. solea in the AmP collection (Teal, 2015). Indeed, (1) we used the newest methods for DEB parameters' calibration based on the covariation method implemented into the DEBtool package, (2) we gathered the most widely-available data from the literature for male and female ever used for this species calibration, including ingestion datasets that have never been considered, and $(3)$ the overall adjustment (MRE $=0.187$, SMSE $=0.173$ ) was slightly better that the one from the previous model using the same kind of estimation procedure (MRE $=0.192$ and SMSE $=0.267$, from "AmP Solea solea version 2015/08/28").

The overall goodness of fit of the present model was even more satisfying given the large diversity of datasets used, which highlighted the robustness of this new model. Indeed, DEB theory assumes that the parameter set is individual-specific. Thus, the estimated parameter set corresponded to that of a mean individual. However, distributions of individual parameter values may depend on the population considered (i.e., different genotypes). Consequently, the mean individual can be different from one dataset to another, whereas only one parameter set was estimated. To deal with that constraint, inflated by the large number of datasets used, we made choices about model adjustment to each dataset according to our modelling applications objectives (see SM 1.1.3 where they are discussed). This calibration strategy led to a set of estimates that permitted to better predict growth inflection compared to the 2015-AmP model.

\section{Modelling sexual growth dimorphism}

As ingestion and growth are two major processes involved in POPs bioaccumulation, and spawning on decontamination, it is important to specify the influence of sex on these different processes (Bodiguel et al., 2009). Thus, the present model explicitly considered sexual dimorphism in modelling sole energy allocation dynamics. We considered both sex as did Eichinger et al. (2010) but we considered a difference in ingestion instead of in the portion of mobilised energy allocated to growth $(\kappa)$. With this assumption, the model permitted to accurately reproduce growth dimorphism observed for female and male in the dataset used for calibration, that was common with the work from Eichinger et al. (2010). Kooijman (2014) assumed that elephant seal (Mirounga leonina) growth dimorphism observed after puberty was induced by sex differentiation of maximum food assimilation rate $\left\{\dot{p}_{A m}\right\}$ only after puberty. Here, as in the Atlantic halibut model, we applied this difference on $\left\{\dot{p}_{A m}\right\}$ throughout the life cycle. It implied that females and males feed and grow differently even before maturity. This assumption was supported by a study on Solea senegalensis, a closely related species, conducted by Viñas et al., (2013), who concluded that this species shows an early gonadal and growth dimorphism (i.e., observable from 3 to 6 months old, histological observations of spermatogonia or oogonia). Moreover, in the present model application on juvenile growth, this assumption led to growth differences between sexes that were included in the observed variability. Thus, we could not reject this assumption using this dataset. Also, in model validation, during larval growth predicted for ad libitum condition, sex differences in length led to a difference of $2 \%$ at $35 \mathrm{dph}$ (i.e., $0.4 \mathrm{~mm}$ ), which is also included in the observed variability at $35 \mathrm{dph}$ (i.e., $0.7 \mathrm{~mm}$ thus $8 \%$ difference between min and max observations).

\section{Puberty stage}

Maturity level at puberty $\left(E_{H}^{p}\right)$ was also a sex-specific parameters. We were able to obtain parameter estimates that had, in the validation step on a North Sea population, a more accurate prediction of the 
length at which females are able to produce maturing oocytes compared to the 2015-AmP model. The error on female age at puberty prediction in the calibration step of the current model (RE $=0.24$, see SM section 1.1.2) is probably due the uncertainty on the data used, as it was inferred from observations of gonadal activity during spawning period (see SM section 1.1.3). Indeed, puberty does not imply immediate gonadal activity for female. Thus, age at puberty data used is often too high. As a consequence, as data on length and weight at puberty were inferred from this age at puberty coupled with age-length or length-weight relationship, respectively, an underestimation of this age induced an underestimation of length, and an even higher effect on weight fit (i.e., due to the strong slope of lengthweight relationship).

\section{Delayed female first reproduction}

The accuracy of the predicted first reproduction depends on $E_{H}^{p}$ value, and on the reproduction rules for female. The latter have been improved to gain realism and to lay the foundations for further research on adults' decontamination through gametes emission, contaminant transfer to next generations, and thus, impact of contamination on population dynamics. It is noteworthy that their full validation is beyond the scope of the present study, as they require further analysis and confrontation to measurements. However, our assumption about the delayed first reproduction due to egg maturation duration was congruent with several previous observations, in addition to those of Ramsay and Witthames (1996). Indeed, Le Bec (1983) also observed that oocyte maturation of the youngest mature soles $(25-30 \mathrm{~cm}$, probably in their first year of maturity) was stopped in vitellogenesis by the end of the spawning period. Bromley (2003) also observed this lack of full-maturation in these individuals, referred as "non-spawning adolescents". They also observed that, after the spawning season, for this year class, the ovaries were fully emptied, with evidence of atresia. Consequently, a portion of the "first year matured females" seems to develop eggs, justifying considering a cost of transformation into gametes, but abort development and reabsorbed these oocytes, justifying considering no spawning for these females. However, some additional research is necessary to support the assumption of fully lost energy through atretic resorption. This delayed first reproduction highlights the need to study the variability in the birth date within the spawning period and in the nursery quality experienced in the context of global changes, as these factors are probably the source of the portion of females from this year class that would not be able to spawn.

\section{Female fecundity}

For the larger females, the present model adjustment to the fecundity dataset from a North Sea population was poorer than that of the 2015-AmP model. However, first, the value of the energy content of one egg $\left(E_{0}\right)$ used for this population was much lower in the 2015-AmP model than in the present-AmP model (i.e., $E_{0}=0.34 \mathrm{~J}$ and $2.54 \mathrm{~J}$, respectively, considering their respective $f$ values). Moreover, the 2015-AmP value for $E_{0}$ was probably unrealistic. Indeed, contrary to the 2015-AmP model, we used information from two different sources of measurements for $E_{0}$ in our calibration. They both indicated an estimate of approximately $2 \mathrm{~J}$ (see SM 1.1.3 - Female egg production). Thus, 2015AmP model was probably able to predict a greater fecundity because the $E_{0}$ value was too low.

In addition, the authors who produced the fecundity dataset used in the 2015-AmP model highlighted that this zone (i.e., IVb East) have the highest fecundity observed, indicating either "phenotypic plasticity or genotypic evolution" (Witthames et al., 1995). As the latter assumption would probably lead to the differentiation of DEB parameters' estimates for this population, it could explain the lack of model fit using parameters estimated for another population. However, the complementary dataset from the same publication but for an adjacent zone from North Sea (i.e., IVb West) was in better agreement with present AmP model predictions. Consequently, the present DEB estimates set for $S$. solea could be applied to different populations, even from the North Sea. However, knowledge of the growth rate and the length-fecundity relationship of the population would reduce the uncertainty in the predictions of the present-AmP model. 


\section{Male reproduction}

Unfortunately, for males, no validation on growth and no quantitative observations of reproduction were found to confront our assumptions about male first reproduction, gametes emission (i.e., multiple batches), and parameters involved only in reproduction $\left(\varrho_{R}\right.$ and $\left.\kappa_{\mathrm{R}}\right)$. However, we considered that the estimation of a 2 to 3 fold lower proportion of energy allocated to reproduction fixed in emitted gametes $\left(\kappa_{\mathrm{R}}\right)$ in male compared to female was plausible. Indeed, semen composition is supposed to be much different from reserve composition, as its composition differs from egg composition, which is assumed to be close the reserve composition. Indeed, contrary to egg, semen contains a poor overall lipid content with a larger portion of polar lipids, which are structural lipids, compared to non-polar lipids, which are reserve lipids (Vassallo-Agius et al., 2001). Thus, it would require more energy for male than for female to transform reserve into gametes. Moreover, spermatogenesis may also require more energy due to the proteins required during meiosis, that may lose its function, which leads to apoptosis for $30 \%$ of the germ cells for the Nile tilapia and $45 \%$ for the Guppy (Schulz et al., 2010).

\section{Early life stages}

The present model permitted also to predict accurately the metamorphosis stage, which is crucial to better predict larvae settlement in nurseries. Yet, in the calibration step, earlier stages are less well predicted than the metamorphosis stage (i.e., regarding ages at stage and their relationship to temperature). However, in both calibration and validation steps, thus in different experimental conditions, the model led to satisfactory predictions of larval growth from birth to metamorphosis. Indeed, considering the range of possible artemia's quality, the only assumption needed for forcings given that the number of artemias ingested was known, both growth in weight and metamorphosis stage observations were comprised between the predictions. For model fit to growth in length, the same trend was observed in calibration and validation steps: length was underestimated the beginning of larval development and overestimated at the end. This can be due to the modelling of shape evolution during larval development and metamorphosis. The refinement of this auxiliary theory to obtain total length from predicted structure may be necessary. However, as growth monitoring may be necessary to study bioaccumulation and toxicity during the larval stage, we recommend the use of weight measurements, which was particularly well predicted by the model.

\subsection{Added value of model application on contamination-decontamination experiment}

The inter-individual variability of contamination in dietary contamination experiments is often large (Eichinger et al., 2010). Consequently, it adds uncertainty on the estimates of toxicokinetic parameters. In the experiment used in this paper, authors also highlighted a large variability in growth. Here, the use of DEB model with different initial weights and levels of food availability allowed to show that feeding variability could lead to both growth and contamination variability.

Variability in feeding conditions was already underlined in a DEB framework by Stavrakidis-Zachou et al. (2019) on European sea bass reared from aquaculture. They reported high temporal variations on the scaled functional response ( $f$ parameter from 0.4 to 1 ) estimated to reproduce mean population growth dynamics. The authors supposed that it was due to "irregularities in the feeding scheme". In the present application we wanted to reproduce inter-individual variability of $f$, probably due to competition for food accessibility (i.e., belt feeder located only in one part of the tank). The modelled variability (i.e., $f$ between 0.2 and 1 ) led to extreme growth curves corresponding to the curves described either by the smallest or the largest fish at each time step. Thus, inter-individual variability seems to be larger in our model application than mean population variability through time for sea bass. Also, considering that CB153 is poorly eliminated by fish (Kannan et al., 1995), it could be used as a feeding tracer. Thus, the good reproduction by the model of growth and contamination variabilities supports our assumptions on $f$ variability and CB153 toxicokinetic properties (i.e., no elimination and a full assimilation from the assimilated part of food). 
As a consequence, individual monitoring of growth, and the knowledge of initial biometry for each fish, may be crucial to better estimate individual feeding thanks to the DEB model in this type of experiment, and thereafter to better estimate toxicokinetic parameters. Moreover, our results seems to highlight that taking into account this feeding variability is as important as taking into account metabolisation to better understand fish contamination. Indeed, when considering $f$ variability, with full assimilation of contaminant from food and no elimination, the model fit for both CB105 as well as for CB153. Yet, contrary to CB153, CB105 can be metabolized by fish (Kannan et al., 1995). Therefore, the feeding variability was probably so large that we were not able to detect that this contaminant is metabolizable.

\section{Conclusion}

Our study allowed improving the common sole DEB model parameterisation and calibration for each sex and several types of environment. These improvements lay solid foundations for future use of this tool in bioaccumulation studies on the juvenile phase, both in contaminated nurseries and in experimental conditions. Indeed, the modelling framework developed here is the first step in the development of a mechanistic TK model that would permit to consider ingestion, growth and contamination variability in different environments, and for different life stages, to be used in a wide range of applications. First, we showed in the present work that taking feeding variability into account is a necessary prerequisite to evaluate toxicokinetic parameters in experimental conditions on juvenile stage. Then, in a second paper from Mounier et al. (2020), we show that this framework was useful for the study of juvenile growth and contamination sensitivity to warming, nursery quality, and prey contamination, in highly fluctuating estuarine environments.

Finally, in a long-term perspective, this modelling framework allows to include contaminant transfer to the next generations and adverse effects from internal concentration (e.g., DEB-tox) on demographic characters (i.e., growth, reproduction, survival of the different life stages). Ultimately, predicted consequences of warming, diet modifications and contamination on these individual characters will allow studying their consequences on population dynamics (Alunno-Bruscia et al., 2009; Horri et al., 2018). 


\section{Acknowledgments}

This study has been carried out with financial support from the French National Research Agency (ANR) in the frame of the Investments for the future Programme, within the Cluster of Excellence COTE (ANR10-LABX-45) as also by the IdEx Bordeaux International Post-doctorates program attributed to A. E. Sardi. Florence Mounier benefited of a PhD grant from INRAE and Ifremer. This study was also partly finalized within the CHOPIN project financed by the Seine Aval program SA6. We also acknowledge Marie-Laure Bégout and Xavier Cousin, from Ifremer, for their expertise on sole larvae experiment.

\section{CRediT authorship contribution statement}

- Mounier F.: Conceptualization, Methodology, Software, Formal analysis, Visualization, Writing - Original Draft, Writing - Review

- Pecquerie L.: Conceptualization, Methodology, Writing - Original Draft

- Lobry J.: Project administration, Funding acquisition, Conceptualization, Methodology, Writing - Original Draft, Writing - Review

- Sardi A. E.: Conceptualization, Methodology, Investigation, Writing - Original Draft

- Labadie P.: Funding acquisition, Project administration, Supervision, Resources, Writing - Original Draft

- Budzinski H.: Funding acquisition, Project administration, Supervision, Resources

- Loizeau V.: Conceptualization, Methodology, Writing - Original Draft 


\section{Appendix: The DEB model and associated equations}

\section{A0. DEB theory and modelling framework}

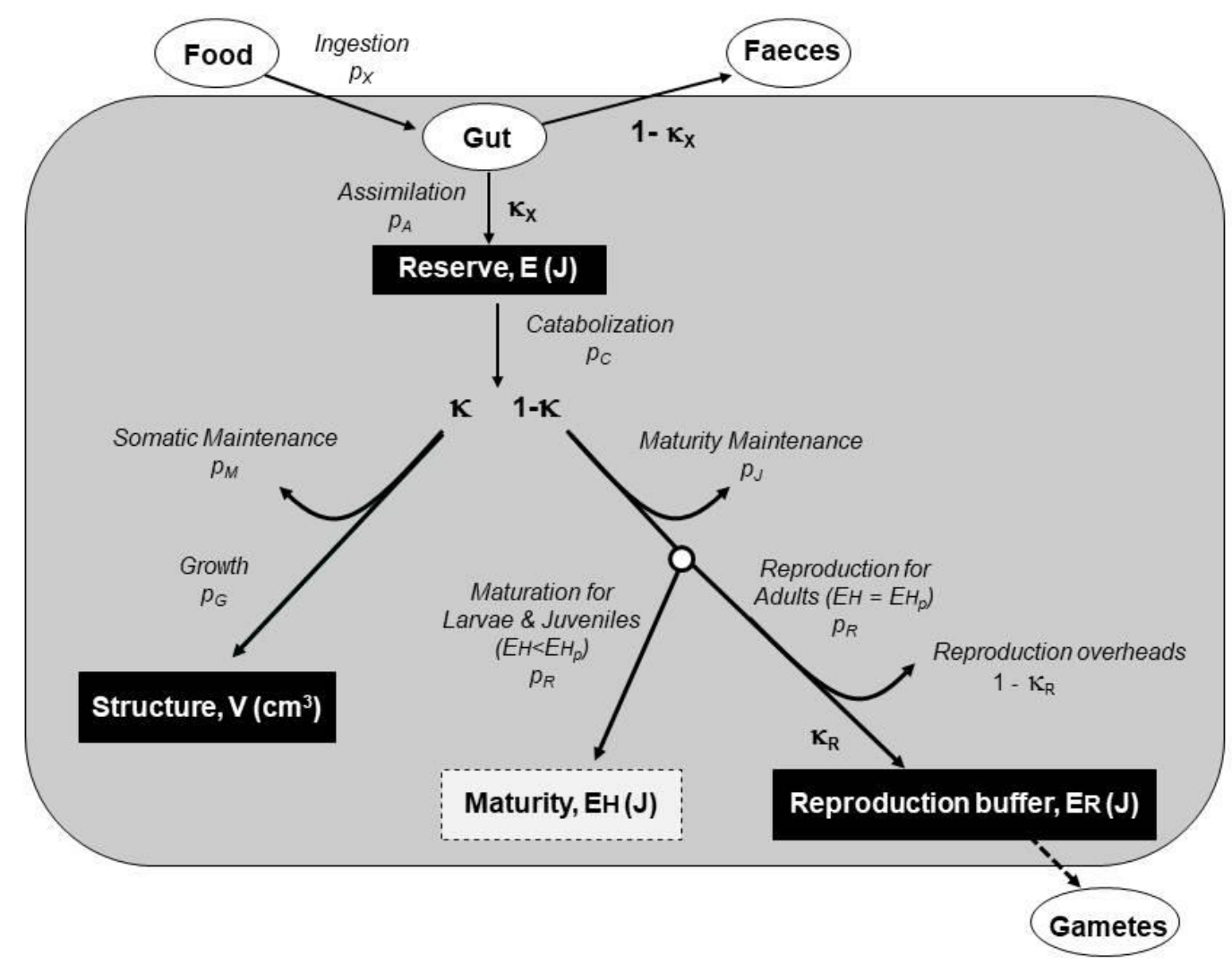

Figure A0 - Schematisation of the DEB model framework used. Arrows are fluxes. Boxes are state variables. Black one are involved in biomass whereas the white dashed one is a virtual compartment. White circles indicate compartments considered as external to the fish. The white node indicates the switch at puberty between maturation and reproduction.

DEB theory assumes that an animal can be modelled by four state variables: reserve, structure, maturity and reproduction. The schematic representation of DEB model framework used is presented in Figure A0. Reserve $(E)$ stores energy inputs from the assimilated portion of food ingested $\left(\dot{p}_{A}=\kappa_{X} \dot{p}_{X}\right)$ and fuels every biological function. A constant proportion $(\kappa)$ of energy mobilized from reserve compartment $\left(\dot{p}_{C}\right)$ is first allocated to structural maintenance $\left(\dot{p}_{M}\right)$ and the remaining energy is dedicated to growth $\left(\dot{p}_{G}\right)$ to be transformed into structural volume $(V)$. The other part of mobilized energy $(1-\kappa)$ is first allocated to maturity maintenance $\left(\dot{p}_{J}\right)$ and the remaining energy is dedicated to the increase of maturity level $\left(E_{H}\right)$ for juveniles or to the reproduction buffer $\left(E_{R}\right)$ for adults (see 2.1.3 for more information on this buffer). The maturity level $\left(E_{H}\right)$ is a virtual compartment (not involved in biomass) recording energy allocated to the complexification of the animal. It is used to trigger the change in stage of development. 


\section{A1. Table of state variables dynamics}

Involved parameters are described in Table 1. DEB fluxes (i.e., $\dot{p}$ ) are presented in A2.

\begin{tabular}{|c|c|c|c|}
\hline \multirow[t]{2}{*}{ State variables } & Differential equation & \multirow[t]{2}{*}{ Unit } & \multirow[t]{2}{*}{ Initial value (egg) } \\
\hline & $\begin{array}{c}\text { Juvenile } E_{H}< \\
E_{H}^{p^{*}}\end{array}$ & & \\
\hline Reserve & $\frac{d E}{d t}=\dot{p}_{A}-\dot{p}_{C}$ & $J$ & $2.217^{\dagger}$ \\
\hline Structural volume & $\frac{d V}{d t}=\frac{\dot{p}_{G}}{\left[E_{G}\right]}$ & $\mathrm{cm}^{3}$ & $10^{-10}$ \\
\hline Maturity level & $\frac{d E_{H}}{d t}=\dot{p}_{R} \quad \frac{d E_{H}}{d t}=0$ & $J$ & 0 \\
\hline Reproduction buffer & $\frac{d E_{R}}{d t}=0 \quad \frac{d E_{R}}{d t}=\kappa_{R *} \dot{p}_{R}$ & $J$ & 0 \\
\hline
\end{tabular}

* sex-specific parameters

† Value from AmP calibration with $f=0.5841$ (same value as the one used for Douarnenez bay population)

\section{A2. Table of DEB fluxes}

Involved parameters are described in Table 1 and in A3 for $s_{\mathcal{M}} . T$ indices indicate temperaturecorrected parameters (see A4). Asterisks refer to sex-specific parameters.

\begin{tabular}{ll}
\hline Energy flux & Formula \\
\hline Ingestion (after birth $\boldsymbol{E}_{\boldsymbol{H}} \geq \boldsymbol{E}_{\boldsymbol{H} \boldsymbol{b}}$ ) & $\dot{p}_{X}=\frac{s_{\mathcal{M}}\left\{\dot{p}_{A m}\right\}_{T *}}{\kappa_{X}} f V^{2 / 3}$ \\
Assimilation (after birth $\boldsymbol{E}_{\boldsymbol{H}} \geq \boldsymbol{E}_{\boldsymbol{H} \boldsymbol{b}}$ ) & $\dot{p}_{A}=\kappa_{X} \dot{p}_{X}$ \\
Catabolic utilization & $\dot{p}_{C}=\frac{\left[E_{G}\right] s_{\mathcal{M}} \dot{\boldsymbol{v}}_{T} V^{-1 / 3}+\left[\dot{p}_{M}\right]_{T}}{\frac{\left[E_{G}\right]}{E}+\frac{\kappa}{V}}$ \\
Somatic maintenance & $\dot{p}_{M}=\left[\dot{p}_{M}\right]_{T} V$ \\
Growth & $\dot{p}_{G}=\kappa \dot{p}_{C}-\dot{p}_{M}$ \\
Maturity maintenance & $\dot{p}_{J}=\dot{k}_{J} E_{H}$ \\
Reproduction/development & $\dot{p}_{R}=(1-\kappa) \dot{p}_{C}-\dot{p}_{J}$ \\
\hline
\end{tabular}




\section{A3. Type $M$ acceleration}

Type $M$ acceleration consists in the progressive increase of maximum food assimilation rate $\left\{\dot{p}_{A m}\right\}$ and energy conductance $\dot{v}$ during this period thanks to their multiplication by an acceleration factor $s_{\mathcal{M}}$. This factor evolves from $s_{\mathcal{M}}=1$ at birth to reach $s_{\mathcal{M}}=L_{j} / L_{b}$ at metamorphosis. Between these two stages, it evolves along with length: $\mathrm{s}_{\mathcal{M}}=\mathrm{L} / \mathrm{L}_{\mathrm{b}}$. L indicates structural length, the cubic root of structural volume $V$.

\section{A4. Temperature correction}

In DEB theory, all metabolic rates can be scaled to body temperature evolution by multiplying primary parameters describing energy rates at a reference temperature by a correction factor.

$$
\dot{p}_{T}=\dot{p}_{\text {Tref }} \times \exp \left(\frac{T_{A}}{T_{r e f}}-\frac{T_{A}}{T}\right) \times \frac{1+\exp \left(\frac{T_{A L}}{T_{r e f}}-\frac{T_{A L}}{T_{L}}\right)+\exp \left(\frac{T_{A H}}{T_{H}}-\frac{T_{A H}}{T_{r e f}}\right)}{1+\exp \left(\frac{T_{A L}}{T}-\frac{T_{A L}}{T_{L}}\right)+\exp \left(\frac{T_{A H}}{T_{H}}-\frac{T_{A H}}{T}\right)}
$$




\section{References}

Alunno-Bruscia, M., van der Veer, H.W., Kooijman, S.A.L.M., 2009. The AquaDEB project (phase I): Analysing the physiological flexibility of aquatic species and connecting physiological diversity to ecological and evolutionary processes by using Dynamic Energy Budgets. J. Sea Res. 62, 43-48. https://doi.org/10.1016/j.seares.2009.07.005

Bodiguel, X., Maury, O., Mellon-Duval, C., Roupsard, F., Le Guellec, A.-M., Loizeau, V., 2009. A dynamic and mechanistic model of PCB bioaccumulation in the European hake (Merluccius merluccius). J. Sea Res. 62, 124-134. https://doi.org/10.1016/j.seares.2009.02.006

Bromley, P.J., 2003. The use of market sampling to generate maturity ogives and to investigate growth, sexual dimorphism and reproductive strategy in central and south-western North Sea sole (Solea solea L.). ICES J. Mar. Sci. 60, 52-65. https://doi.org/10.1006/jmsc.2002.1318

Budzinski, H., Jones, I., Bellocq, J., Piérard, C., Garrigues, P., 1997. Evaluation of sediment contamination by polycyclic aromatic hydrocarbons in the Gironde estuary. Mar. Chem. 58, 85-97. https://doi.org/10.1016/S0304-4203(97)00028-5

Burreau, S., Axelman, J., Broman, D., Jakobsson, E., 1997. Dietary uptake in pike (Esox lucius) of some polychlorinated biphenyls, polychlorinated naphthalenes and polybrominated diphenyl ethers administered in natural diet. Environ. Toxicol. Chem. 16, 2508-2513. https://doi.org/10.1002/etc.5620161211

Courrat, A., Lobry, J., Nicolas, D., Laffargue, P., Amara, R., Lepage, M., Girardin, M., Le Pape, O., 2009. Anthropogenic disturbance on nursery function of estuarine areas for marine species. Estuar. Coast. Shelf Sci. 81, 179-190. https://doi.org/10.1016/j.ecss.2008.10.017

Daouk, T., Larcher, T., Roupsard, F., Lyphout, L., Rigaud, C., Ledevin, M., Loizeau, V., Cousin, X., 2011. Long-term food-exposure of zebrafish to PCB mixtures mimicking some environmental situations induces ovary pathology and impairs reproduction ability. Aquat. Toxicol. 105, 270-278. https://doi.org/10.1016/j.aquatox.2011.06.021

Day, O.J., Jones, D.A., Howell, B.R., 1996. Food consumption, growth and respiration of sole, Solea solea (L.), during early ontogeny in a hatchery environment. Aquac. Res. 27, 831-839. https://doi.org/10.1046/j.1365-2109.1996.t01-1-00800.x

Delpech, C., Courrat, A., Pasquaud, S., Lobry, J., Le Pape, O., Nicolas, D., Boët, P., Girardin, M., Lepage, M., 2010. Development of a fish-based index to assess the ecological quality of transitional waters: The case of French estuaries. Mar. Pollut. Bull. 60, 908-918.

Deniel, C., 1981. Les poissons plats [Téléostéens, Plueornectiformes] en baie de Douarnenez: reproduction, croissance et migration. J. Fish Biol. Université de Bretagne Occidentale - Brest.

Devauchelle, N., Alexandre, J.C., Corre, N.L.E., Letty, Y., 1987. Spawning of Sole (Solea solea) in Captivity. Aquaculture 66, 125-147.

Dorel, D., 1986. Poissons de l'Atlantique Nord-Est, Relations Taille-Poids. Nantes.

Eichinger, M., Loizeau, V., Roupsard, F., Le Guellec, A.-M., Bacher, C., 2010. Modelling growth and bioaccumulation of Polychlorinated biphenyls in common sole (Solea solea). J. Sea Res. 64, 373385. https://doi.org/10.1016/j.seares.2010.05.005

El-Shahawi, M.S., Hamza, a, Bashammakh, a S., Al-Saggaf, W.T., 2010. An overview on the accumulation, distribution, transformations, toxicity and analytical methods for the monitoring of 
persistent organic pollutants. Talanta 80, 1587-97. https://doi.org/10.1016/j.talanta.2009.09.055

Fisk, A.T., Norstrom, R.J., Cymbalisty, C.D., Muir, D.G.G., 1998. Dietary accumulation and depuration of hydrophobic organochlorines: Bioaccumulation parameters and their relationship with the octanol/water partition coefficient. Environ. Toxicol. Chem. 17, 951-961. https://doi.org/10.1897/1551-5028(1998)017<0951:DAADOH>2.3.CO;2

Fonds, M., 1979. Laboratory Observations on the Influence of Temperature and Salinity on Development of the Eggs and Growth of the Larvae of Solea solea (Pisces). Mar. Ecol. Prog. Ser. 1, 91-99. https://doi.org/10.3354/meps001091

Fonds, M., Drinkwaard, B., Resink, J.W., Eysink, G.G.J., Toet, W., 1989. Measurements of metabolism, food intake and growth of Solea solea (L.) fed with mussel meat or with dry food, in: Aquaculture: A Biotechnology in Progress: Volume 2. pp. 851-874.

Fonds, M., Saksena, V.P., 1977. The daily food intake of young soles (Solea solea L,) in relation to their size and the water temperature. I.C.E.S. Work. Gr. Maric. 4, 51-58.

Fonseca, V.F., Vasconcelos, R.P., França, S., Serafim, A., Lopes, B., Company, R., Bebianno, M.J., Costa, M.J., Cabral, H.N., 2014. Modeling fish biological responses to contaminants and natural variability in estuaries. Mar. Environ. Res. 96, 45-55. https://doi.org/10.1016/j.marenvres.2013.10.011

Freitas, V., Cardoso, J.F.M.F., Lika, K., Peck, M.A., Campos, J., Kooijman, S.A.L.M., van der Veer, H.W., 2010. Temperature tolerance and energetics: a dynamic energy budget-based comparison of North Atlantic marine species. Philos. Trans. R. Soc. B Biol. Sci. 365, 3553-3565. https://doi.org/10.1098/rstb.2010.0049

Galois, R., Lagardere, F., Richard, P., 1990. Changes in biochemical composition and otolith microstructure of larval common soleas, Solea solea (L.) under experimental starvation. La Mer.

Gibson, R.N., 1994. Impact of habitat quality and quantity on the recruitment of juvenile flatfishes. Netherlands J. Sea Res. 32, 191-206. https://doi.org/10.1016/0077-7579(94)90040-X

Gilliers, C., Le Pape, O., Désaunay, Y., Morin, J., Guérault, D., Amara, R., 2006. Are growth and density quantitative indicators of essential fish habitat quality? An application to the common sole Solea solea nursery grounds. Estuar. Coast. Shelf Sci. 69, 96-106. https://doi.org/10.1016/j.ecss.2006.02.006

Gobas, F.A.P.C., Muir, D.C.G., Mackay, D., 1988. Dynamics of dietary bioaccumulation and faecal elimination of hydrophobic organic chemicals in fish. Chemosphere 17, 943-962. https://doi.org/10.1016/0045-6535(88)90066-5

Hoegh-Guldberg, O., Bruno, J.F., 2010. The impact of climate change on the world's marine ecosystems. Science 328, 1523-1528. https://doi.org/10.1126/science.1189930

Horri, K., Alfonso, S., Cousin, X., Munschy, C., Loizeau, V., Aroua, S., Bégout, M.L., Ernande, B., 2018. Fish life-history traits are affected after chronic dietary exposure to an environmentally realistic marine mixture of PCBs and PBDEs. Sci. Total Environ. 610-611, 531-545. https://doi.org/10.1016/j.scitotenv.2017.08.083

IPBES, 2019. Global assessment report on biodiversity and ecosystem services of the Intergovernmental Science-Policy Platform on Biodiversity and Ecosystem Services.

Kannan, N., Reusch, T.B.H., Schulz-Bull, D.E., Petrick, G., Duinker, J.C., 1995. Chlorobiphenyls: Model Compounds for Metabolism in Food Chain Organisms and Their Potential Use as Ecotoxicological 
Stress Indicators by Application of the Metabolic Slope Concept. Environ. Sci. Technol. 29, 18511859. https://doi.org/10.1021/es00007a024

Kooijman, S.A.L.M., 2014. Metabolic acceleration in animal ontogeny: An evolutionary perspective. J. Sea Res. 94, 128-137. https://doi.org/10.1016/j.seares.2014.06.005

Kooijman, S.A.L.M., 2010. Dynamic Energy Budget Theory for Metabolic Organisation - Third edition. https://doi.org/https://doi.org/10.1017/СBO9780511805400

Kooijman, S.A.L.M., 2000. Dynamic Energy and Mass Budgets in Biological Systems. Respiration 2, pp 424. https://doi.org/10.1017/CBO9780511565403

Le Bec, C., 1983. Cycle sexuel et fécondité de la sole Solea vulgaris (Quensel, 1806) du Golfe de Gascogne. Rev. des Trav. I'Institut des Pêches Marit. 47, 179-189.

Le Pape, O., Holley, J., Guérault, D., Désaunay, Y., 2003. Quality of coastal and estuarine essential fish habitats: estimations based on the size of juvenile common sole (Solea solea L.). Estuar. Coast. Shelf Sci. 58, 793-803. https://doi.org/10.1016/S0272-7714(03)00185-9

Lika, K., Augustine, S., Pecquerie, L., Kooijman, S.A.L.M., 2014. The bijection from data to parameter space with the standard DEB model quantifies the supply-demand spectrum. J. Theor. Biol. 354, 35-47. https://doi.org/10.1016/j.jtbi.2014.03.025

Lika, K., Kearney, M.R., Freitas, V., van der Veer, H.W., van der Meer, J., Wijsman, J.W.M., Pecquerie, L., Kooijman, S.A.L.M., 2011. The "covariation method" for estimating the parameters of the standard Dynamic Energy Budget model I: Philosophy and approach. J. Sea Res. https://doi.org/10.1016/j.seares.2011.07.010

Liu, J., Haffner, G.D., Drouillard, K.G., 2010. The influence of diet on the assimilation efficiency of 47 polychlorinated biphenyl congeners in Japanese koi (Cyprinus carpio). Environ. Toxicol. Chem. 29, 401-409. https://doi.org/10.1002/etc.47

Lund, I., Steenfeldt, S.J., Suhr, K.I., Hansen, B.W., 2008. A comparison of fatty acid composition and quality aspects of eggs and larvae from cultured and wild broodstock of common sole (Solea solea L.). Aquac. Nutr. 14, 544-555. https://doi.org/10.1111/j.1365-2095.2007.00560.x

Mackay, D., Fraser, A., 2000. Bioaccumulation of persistent organic chemicals: mechanisms and models. Environ. Pollut. 110, 375-391. https://doi.org/10.1016/S0269-7491(00)00162-7

Marques, G.M., Augustine, S., Lika, K., Pecquerie, L., Domingos, T., Kooijman, S.A.L.M., 2018a. The AmP project: Comparing species on the basis of dynamic energy budget parameters. PLOS Comput. Biol. 14, e1006100. https://doi.org/10.1371/journal.pcbi.1006100

Marques, G.M., Lika, K., Augustine, S., Pecquerie, L., Kooijman, S.A.L.M., 2018b. Fitting multiple models to multiple data sets. J. Sea Res. 0-1. https://doi.org/10.1016/j.seares.2018.07.004

Matthiessen, P., Law, R.J., 2002. Contaminants and their effects on estuarine and coastal organisms in the United Kingdom in the late twentieth century. Environ. Pollut. 120, 739-757. https://doi.org/10.1016/S0269-7491(02)00175-6

Mounier, F., Loizeau, V., Pecquerie, L., Drouineau, H., Labadie, P., 2020. Dietary bioaccumulation of persistent organic pollutants in the common sole Solea solea in the context of global change. Part 2 : Sensitivity of juvenile growth and contamination to toxicokinetic parameters uncertainty and environmental conditions variab. Ecol. Modell. 431, 109196. https://doi.org/10.1016/j.ecolmodel.2020.109196 
Munschy, C., Bely, N., Héas-Moisan, K., Olivier, N., Loizeau, V., 2017. Tissue-specific distribution and maternal transfer of polybrominated diphenyl ethers (PBDEs) and their metabolites in adult common sole (Solea solea L.) over an entire reproduction cycle. Ecotoxicol. Environ. Saf. 145, 457465. https://doi.org/10.1016/j.ecoenv.2017.07.062

Nunes, M., Martinho, F., Vernisseau, A., Marchand, P., Le Bizec, B., van der Veer, H.W., Cabral, H.N., Ramos, F., Pardal, M.A., 2014. Early contamination of European flounder (Platichthys flesus) by PCDD/Fs and dioxin-like PCBs in European waters. Mar. Pollut. Bull. 85, 292-296. https://doi.org/10.1016/j.marpolbul.2014.05.042

Pasquaud, S., Courrat, A., Fonseca, V.F., Gamito, R., Gonçalves, C.I., Lobry, J., Lepage, M., Costa, M.J., Cabral, H., 2013. Strength and time lag of relationships between human pressures and fish-based metrics used to assess ecological quality of estuarine systems. Estuar. Coast. Shelf Sci. 134, 119127. https://doi.org/10.1016/j.ecss.2013.02.002

R core team, 2017. R: A language and environment for statistical computing. R Found. Stat. Comput. Vienna, Austria. https://doi.org/http://www.R-project.org/

Ramsay, K., Witthames, P., 1996. Using oocyte size to assess seasonal ovarian development in Solea solea (L.). J. Sea Res. 36, 275-283. https://doi.org/10.1016/S1385-1101(96)90796-0

Schulz, R.W., de França, L.R., Lareyre, J.J., LeGac, F., Chiarini-Garcia, H., Nobrega, R.H., Miura, T., 2010. Spermatogenesis in fish. Gen. Comp. Endocrinol. 165, 390-411. https://doi.org/10.1016/j.ygcen.2009.02.013

Stavrakidis-Zachou, O., Papandroulakis, N., Lika, K., 2019. A DEB model for European sea bass (Dicentrarchus labrax): Parameterisation and application in aquaculture. J. Sea Res. 143, 262-271. https://doi.org/10.1016/j.seares.2018.05.008

Teal, L.R., 2015. AmP Solea solea version 2015/08/28. Add-my-Pet. http://www.bio.vu.nl/thb/deb/deblab/add_my_pet/entries_web/Solea_solea/Solea_solea_res.h $\mathrm{tml}$

Teal, L.R., van Hal, R., van Kooten, T., Ruardij, P., Rijnsdorp, A.D., 2012. Bio-energetics underpins the spatial response of North Sea plaice (Pleuronectes platessa L.) and sole (Solea solea L.) to climate change. Glob. Chang. Biol. 18, 3291-3305. https://doi.org/10.1111/j.1365-2486.2012.02795.x

Teichert, N., Borja, A., Chust, G., Uriarte, A., Lepage, M., 2016. Restoring fish ecological quality in estuaries: Implication of interactive and cumulative effects among anthropogenic stressors. Sci. Total Environ. 542, 383-393. https://doi.org/10.1016/j.scitotenv.2015.10.068

van der Oost, R., Beyer, J., Vermeulen, N.P.E., 2003. Fish bioaccumulation and biomarkers in environmental risk assessment: A review. Environ. Toxicol. Pharmacol. 13, 57-149. https://doi.org/10.1016/S1382-6689(02)00126-6

van der Veer, H.W., Kooijman, S.A.L.M., van der Meer, J., 2001. Intra- and interspecies comparison of energy flow in North Atlantic flatfish species by means of dynamic energy budgets. J. Sea Res. 45, 303-320. https://doi.org/10.1016/S1385-1101(01)00061-2

Vanhaecke, P., Lavens, P., Sorgeloos, P., 1983. International study on Artemia. XVII. Energy consumption in cysts and early larval stages of varioous geographical strains of Artemia. Annls Soc. r. zool. Belg. $113,155-164$.

Vassallo-Agius, R., Watanabe, T., Yoshizaki, G., Satoh, S., Takeuchi, Y., 2001. Quality of eggs and spermatozoa of rainbow trout fed an n-3 essential fatty acid-deficient diet and its effects on the 
lipid and fatty acid components of eggs, semen and livers. Fish. Sci. 67, 818-827. https://doi.org/10.1046/j.1444-2906.2001.00328.x

Viñas, J., Asensio, E., Cañavate, J.P., Piferrer, F., 2013. Gonadal sex differentiation in the Senegalese sole (Solea senegalensis) and first data on the experimental manipulation of its sex ratios. Aquaculture 384-387, 74-81. https://doi.org/10.1016/j.aquaculture.2012.12.012

Wiedmann, M.A., Augustine, S., Kooijman, S.A.L.M., 2017. AmP Hippoglossus hippoglossus version 2017/03/06.

Add-my-Pet. http://www.bio.vu.nl/thb/deb/deblab/add_my_pet/entries_web/Hippoglossus_hippoglossus/Hi ppoglossus_hippoglossus_res.html.

Witthames, P.R., Greer Walker, M., Dinis, M.T., Whiting, C.L., 1995. The geographical variation in the potential annual fecundity of dover sole Solea solea (L.) from European shelf waters during 1991. Netherlands J. Sea Res. 34, 45-58. https://doi.org/10.1016/0077-7579(95)90013-6 


\section{SUPPLEMENTARY MATERIAL}

For:

Dietary bioaccumulation of persistent organic pollutants in the common sole Solea solea in the context of global change. Part 1: Revisiting parameterisation and calibration of a DEB model to consider inter-individual variability in experimental and natural conditions.

\section{Content}

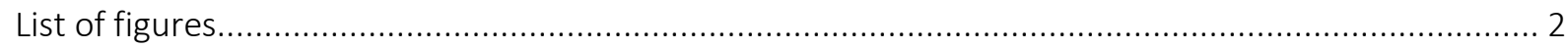

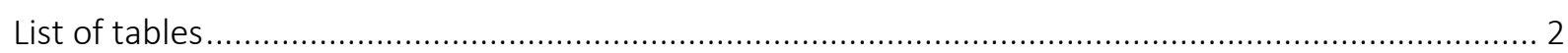

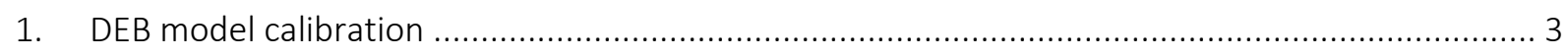

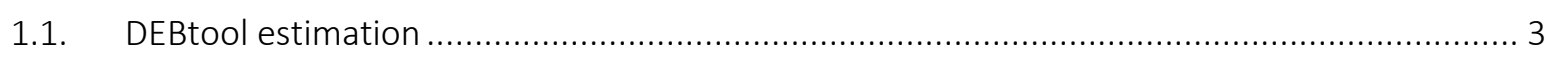

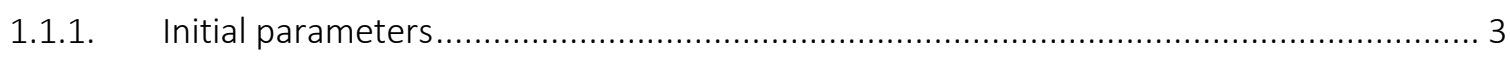

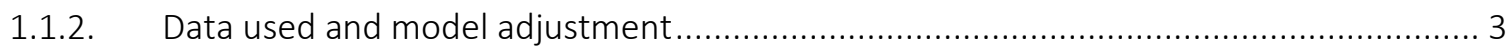

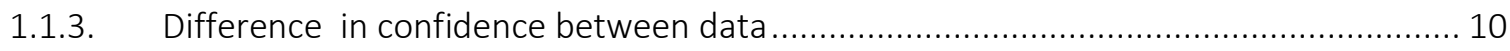

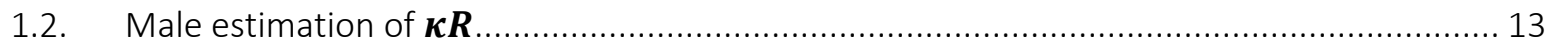

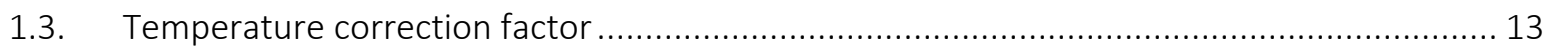

2. Material and methods and results for experimental datasets on larval stage ............................. 15

2.1. Experimental set-up to house common sole larvae in 24-well plates.................................. 15

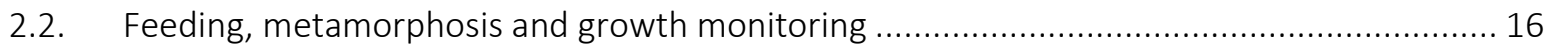

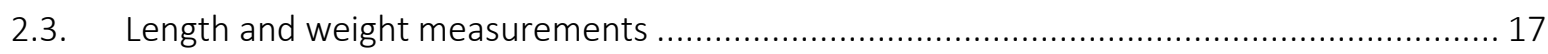

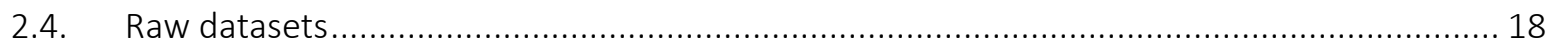

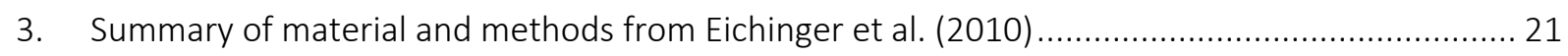

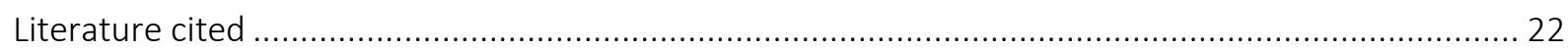




\section{List of figures}

Figure S1 - Annual egg predictions from DEBtool estimation from daily egg production with a constant length all over the year.

Figure S2 - Annual egg predictions from gametes buffer at length divided by the energy content of one egg.

Figure S3 - Temperature evolution of temperature correction factor from our model and the two references used.

\section{List of tables}

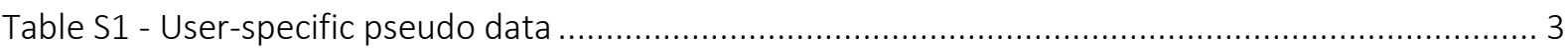

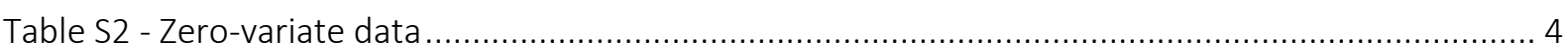

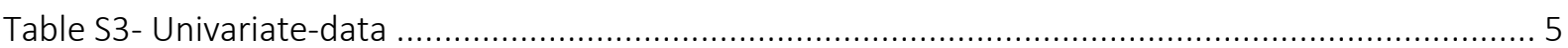

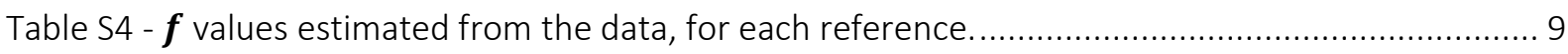

Table S5 - Temperature correction parameters estimates from our model and the two references used.

Table S6 - Chronology of the experimental conditions for rearing larvae in 24-well microplates.

Table S7 - Individual daily food ingestion of larvae in number of artemias adapted for simulation purpose (highlighted columns correspond to the estimations for non feeding days).

Table S8 - Individual daily proportion of food distributed having been ingested for each larva in percent (highlighted columns correspond to the estimations for non feeding days) .......................... 19

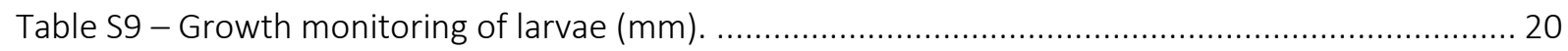

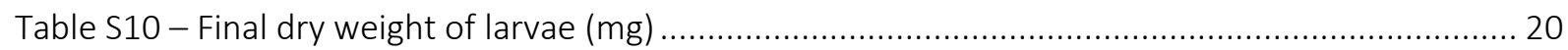




\section{DEB model calibration}

\subsection{DEBtool estimation}

Matlab ${ }^{\circledR}$ files used for the DEBtool estimation are available as Supporting Information files. They contain all code and data used.

\subsubsection{Initial parameters}

Initial parameter values were initially set to parameter values for a generalized animal species (Lika et al., 2011) which are default values in the empty template for 'abj' model from the DEBtool package. Then, we followed the nine steps procedure described in the "Wiki style AmP manual" to manually set initial estimates for parameters of standard model.

\subsubsection{Data used and model adjustment}

Where no information is given about sex (e.g. larval or juvenile data), female parameter set was used.

Table S1 - User-specific pseudo data

\begin{tabular}{|c|c|c|c|c|c|c|}
\hline Symbol & Unit & Data & Prediction & RE & Description & Reference \\
\hline \multirow{6}{*}{ pAm_postj } & \multirow{6}{*}{$\mathrm{J} / \mathrm{d} . \mathrm{cm}^{2}$} & \multirow{6}{*}{800} & \multirow{6}{*}{710.4} & \multirow{6}{*}{0.112} & $\{p A m\}$ after acceleration & \multirow{6}{*}{ Fonds 1989} \\
\hline & & & & & approximated from experiment of daily food ingestion $\left(Q_{X}\right)$ at $20^{\circ} \mathrm{C}$ (Tref) for juvenile soles fed & \\
\hline & & & & & with mussel meat: $\left\{\dot{p}_{A m}\right\}=\left(\kappa_{X} Q_{X} \varrho_{X}\right) /\left(f\left(\delta_{M} L_{w}\right)^{2}\right)$ with assumptions of: & \\
\hline & & & & & $\delta_{M}=0.192$ and $\kappa_{X}=0.8$ (van der Veer et al., 2001) & \\
\hline & & & & & $\varrho_{X}=18100 \mathrm{~J} / \mathrm{g}$ in winter and $19400 \mathrm{~J} / \mathrm{g}$ in summer (Fonds et al., 1989) & \\
\hline & & & & & and $f=1$ & \\
\hline
\end{tabular}


Table S2 - Zero-variate data

\begin{tabular}{|c|c|c|c|c|c|c|}
\hline Symbol & Unit & Data & Prediction & RE & Description & Reference \\
\hline WdO & $\mathrm{mg}$ & 0.12 & 0.09634 & 0.1972 & dry weight of an egg & Devauchelle 1987 \\
\hline EO & J & 2 & 2.217 & 0.1085 & reserve energy of an egg & Devauchelle 1987 \\
\hline ah & $d$ & 4.5 & 6.993 & 0.5541 & age at hatching & Fonds 1979 \\
\hline Lh & $\mathrm{cm}$ & 0.325 & 0.2394 & 0.2633 & total length at hatching & Fonds 1979 \\
\hline Wdh & $\mu \mathrm{g}$ & 51.9 & 28.23 & 0.4561 & dry weight at hatching & Day 1996 \\
\hline$a b$ & $d$ & 8 & 11.24 & 0.4048 & age at birth & Fonds 1979 \\
\hline$W d b$ & $\mu \mathrm{g}$ & 91.96 & 77.13 & 0.1613 & dry weight at birth & Day 1996 \\
\hline tj & $d$ & 28 & 21.36 & 0.2372 & time from birth to metamorphosis & Fonds 1979 \\
\hline $\mathrm{Lj}$ & $\mathrm{cm}$ & 1 & 0.8972 & 0.1028 & total length at the end of metamorphosis & Fonds 1979 \\
\hline Wdj & $\mathrm{mg}$ & 1.8 & 1.759 & 0.02293 & dry weight at metamorphosis & Galois 1990 \\
\hline ap & $d$ & 941.7 & 713.4 & 0.2424 & age at puberty for female & Deniel 1981 \\
\hline Lp & $\mathrm{cm}$ & 27 & 22.51 & 0.1664 & total length at puberty for female & Deniel 1981 \\
\hline ap_m & $d$ & 708.1 & 653.2 & 0.07753 & age at puberty for male & Dorel 1986 \\
\hline Lp_m & $\mathrm{cm}$ & 22 & 20.31 & 0.0766 & total length at puberty for male & Dorel 1986 \\
\hline Wwp_m & g & 87 & 77.95 & 0.104 & wet weight at puberty for male & Deniel 1981 \\
\hline $\mathrm{Li}$ & $\mathrm{cm}$ & 48 & 47.57 & 0.008948 & ultimate total length for female & Deniel 1981 \\
\hline Li_m & $\mathrm{cm}$ & 42 & 44.97 & 0.0706 & ultimate total length for male & Deniel 1981 \\
\hline Wwi & g & 1110 & 1021 & 0.08011 & ultimate wet weight for female & Deniel 1981 \\
\hline Wwi_m & g & 701 & 845.3 & 0.2059 & ultimate wet weight for male & Deniel 1981 \\
\hline am & $d$ & 9490 & 9810 & 0.03375 & life span for female & Deniel 1981 \\
\hline am_m & $d$ & 8760 & 8507 & 0.02888 & life span for male & Deniel 1981 \\
\hline $\mathrm{Ri}$ & $\# / d$ & 3027 & 1821 & 0.3985 & maximum reprod rate per day & Deniel 1981 \\
\hline
\end{tabular}


Table S3- Univariate-data

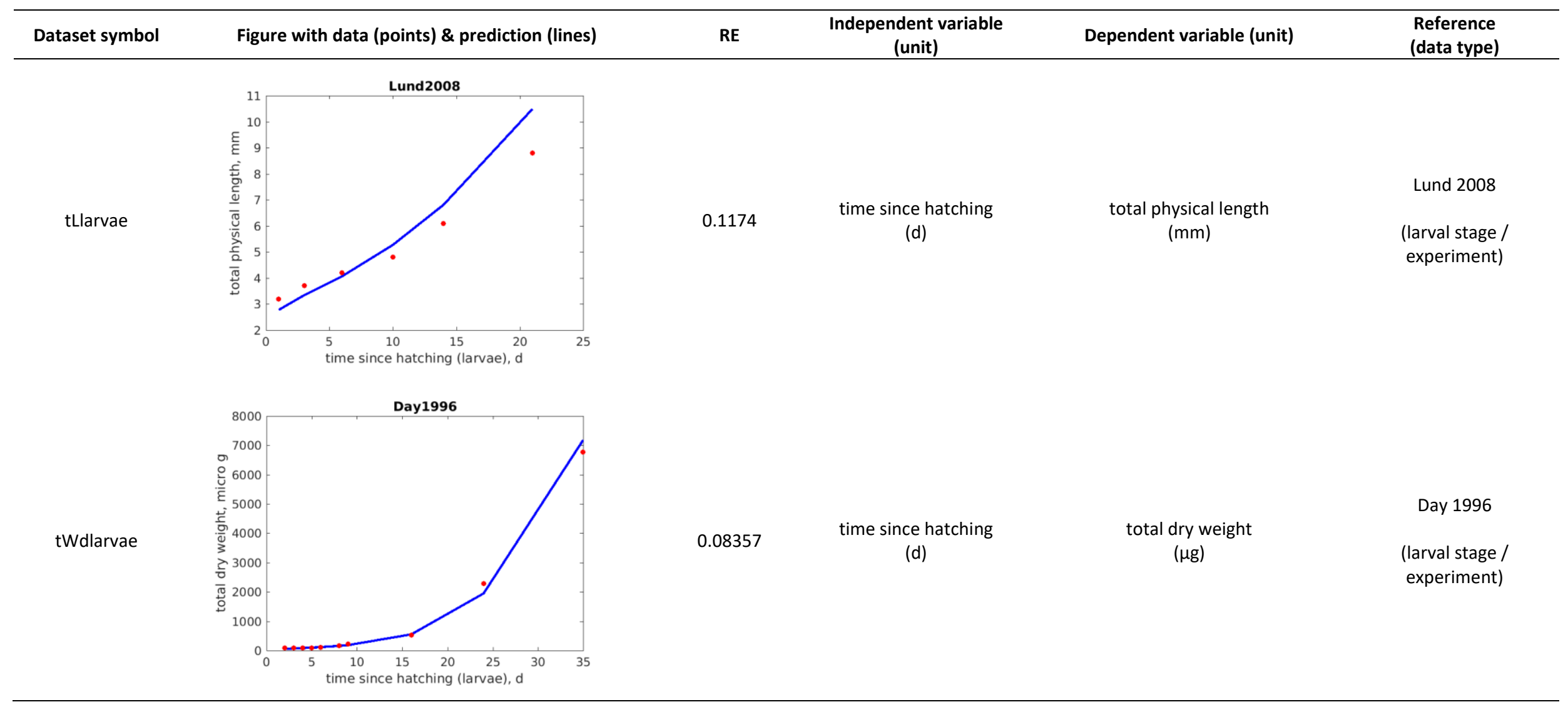




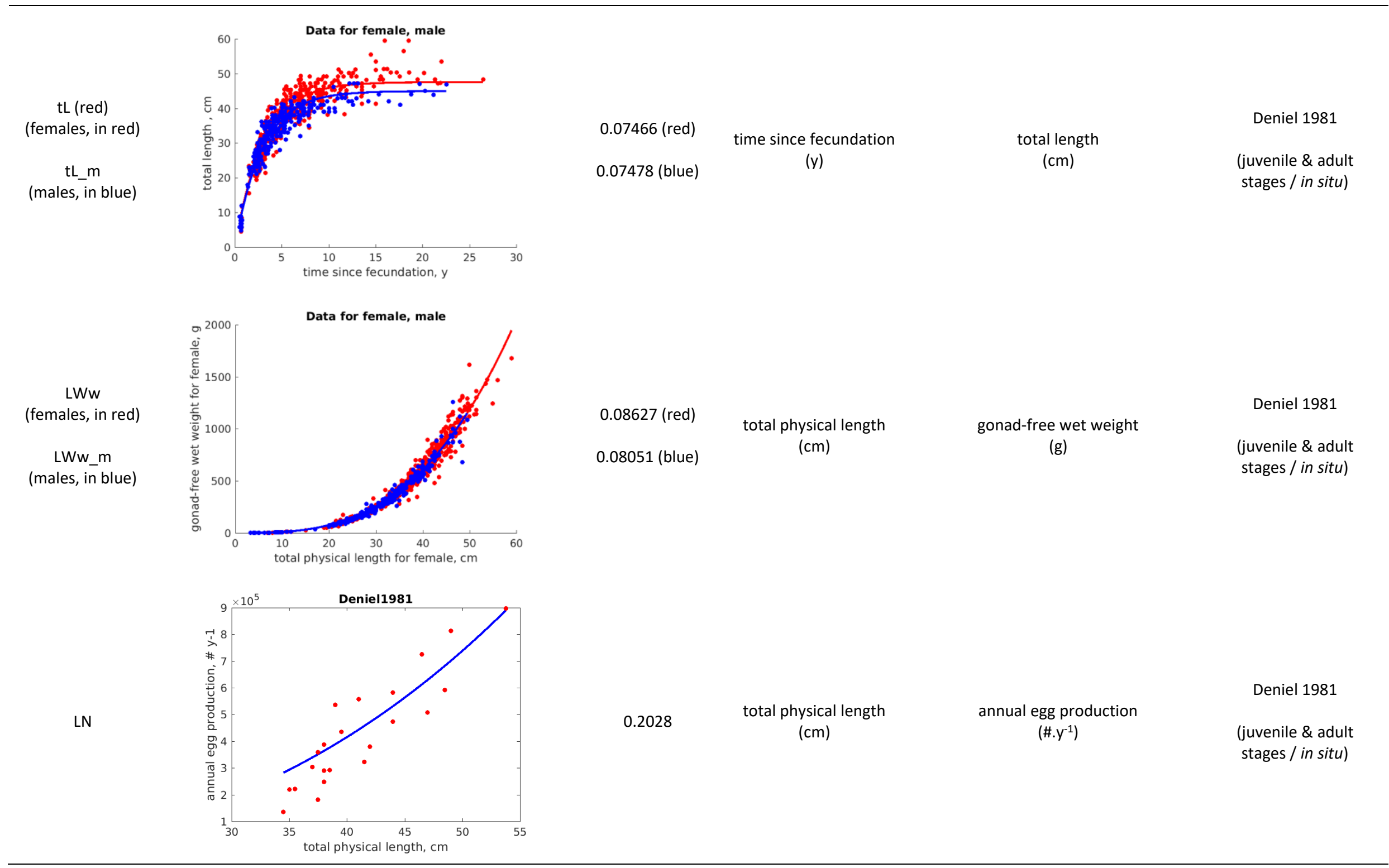


Tah

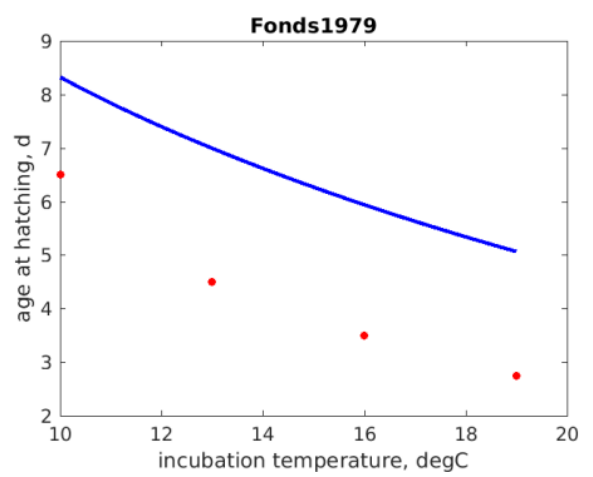

Tab

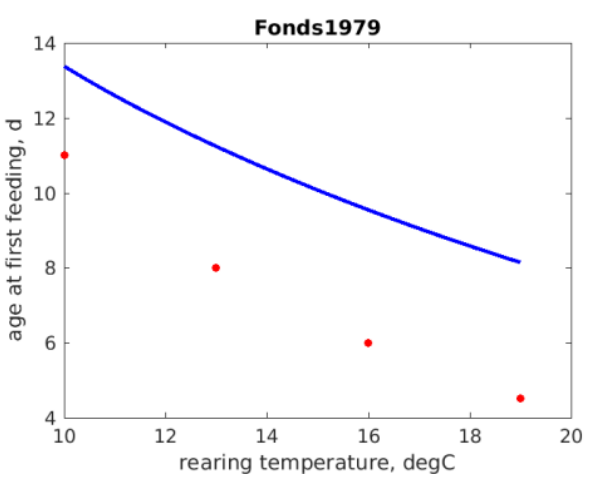

Ttbj

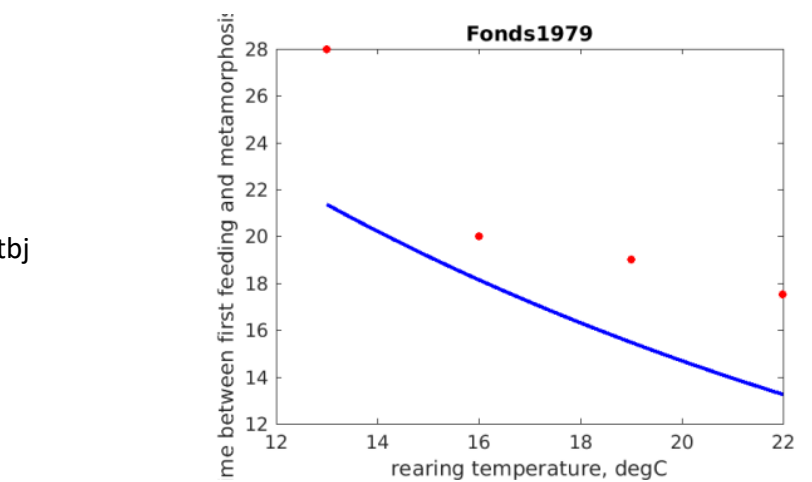

incubation temperature

$$
\left({ }^{\circ} \mathrm{C}\right)
$$

age at hatching
(d)
Fonds 1979

(embryo stage / experiment) rearing temperature

$\left({ }^{\circ} \mathrm{C}\right)$

age at first feeding
(d)
Fonds 1979

(larval stage / experiment)
0.1928 rearing temperature

$$
\left({ }^{\circ} \mathrm{C}\right)
$$

Time between first feeding and metamorphosis
Fonds 1979

(larval stage / experiment) 


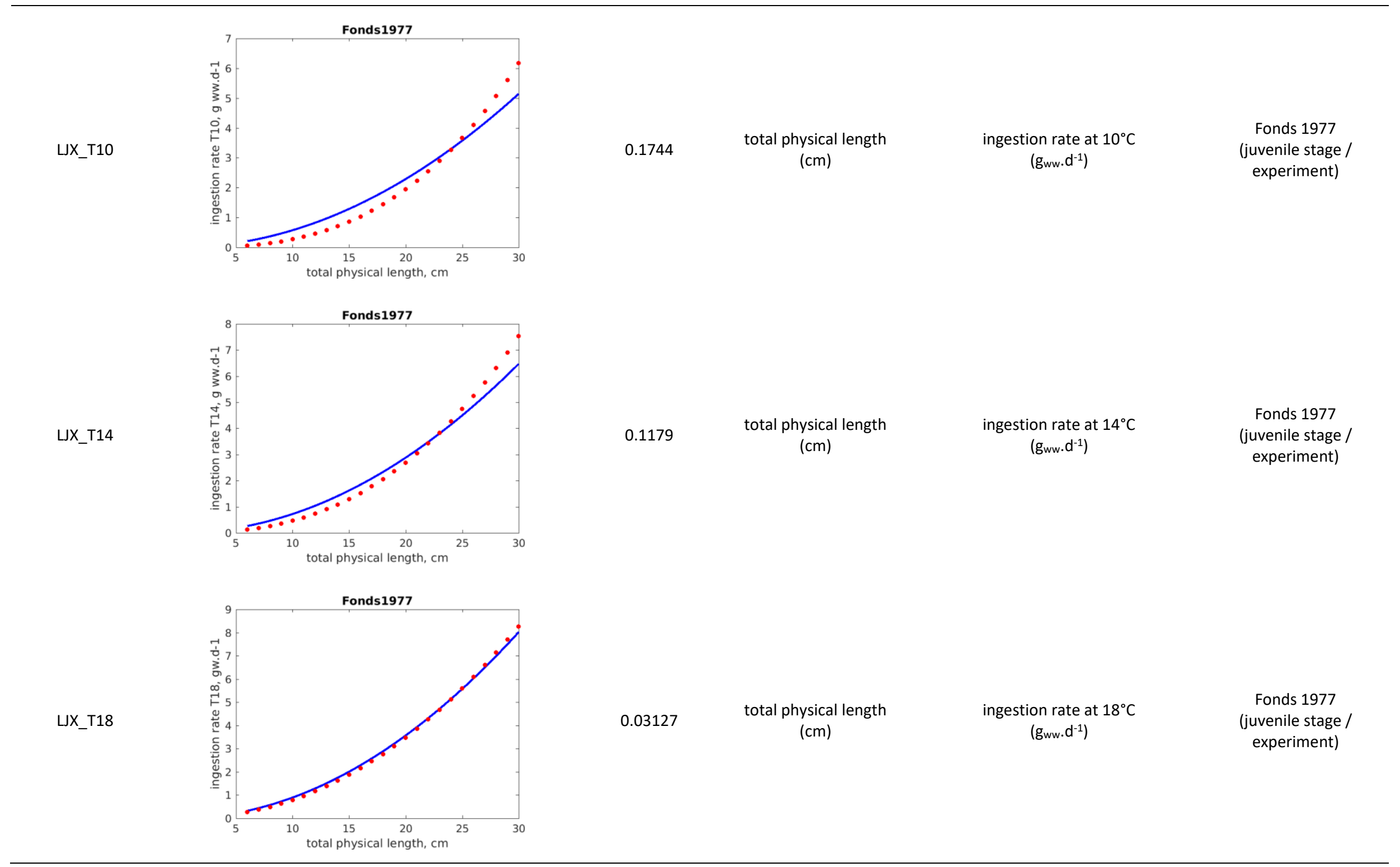




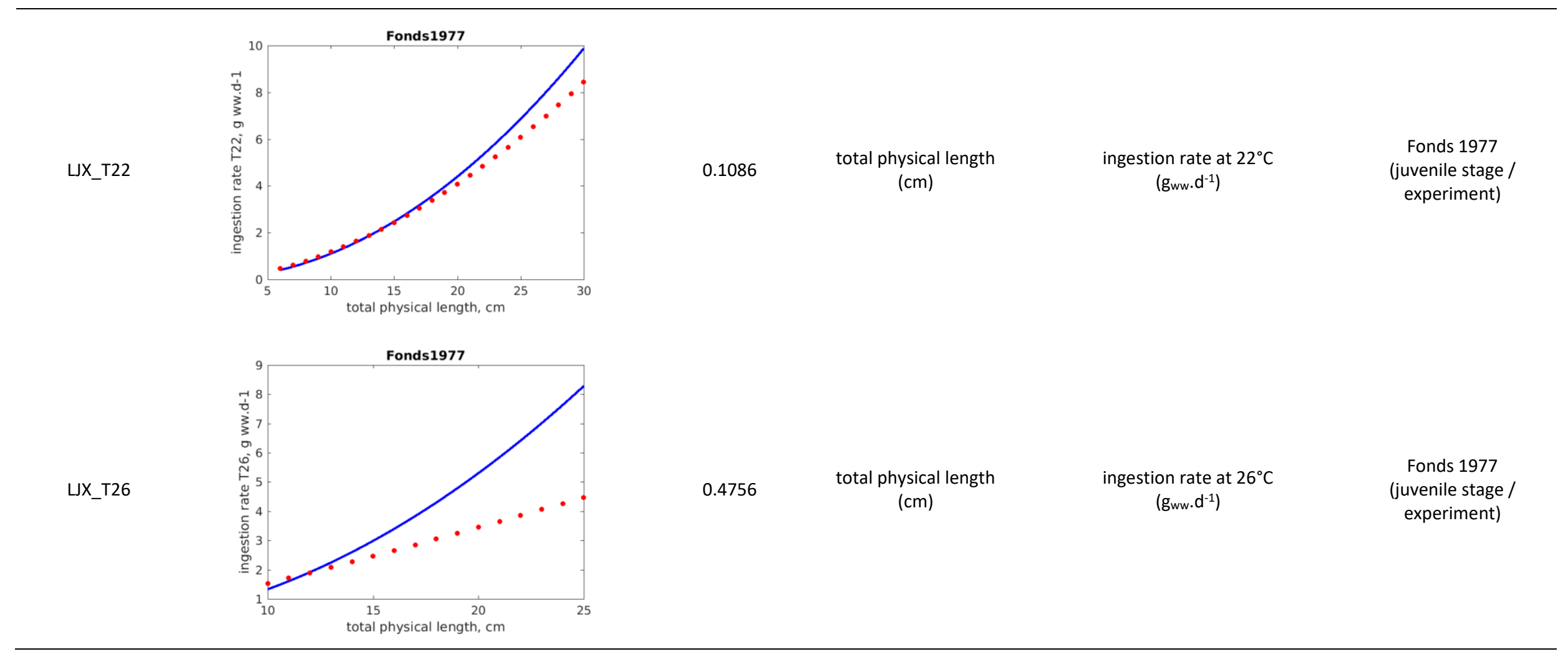

Table S4 - $\boldsymbol{f}$ values estimated from the data, for each reference.

\begin{tabular}{|c|c|c|c|c|c|c|c|}
\hline $\begin{array}{c}\text { Reference } \\
\text { (data type) }\end{array}$ & $\begin{array}{c}\text { Lund } 2008 \\
\text { (larval stage / } \\
\text { experiment) }\end{array}$ & $\begin{array}{c}\text { Day } 1996 \\
\text { (larval stage / } \\
\text { experiment) }\end{array}$ & $\begin{array}{c}\text { Galois } 1990 \\
\text { (larval stage / } \\
\text { experiment) }\end{array}$ & $\begin{array}{c}\text { Fonds } 1979 \\
\text { (larval stage / } \\
\text { experiment) }\end{array}$ & $\begin{array}{c}\text { Fonds } 1977 \\
\text { (juvenile stage / } \\
\text { experiment) }\end{array}$ & $\begin{array}{c}\text { Deniel } 1981 \\
\text { (juvenile \& adult } \\
\text { stages / in situ) }\end{array}$ & $\begin{array}{l}\text { Devauchelle 1987 } \\
\text { (eggs from in situ } \\
\text { genitors) }\end{array}$ \\
\hline$f$ value & 0.5835 & 0.5855 & 0.6778 & 0.5837 & 1.432 & 0.5841 & 0.5841 \\
\hline
\end{tabular}




\subsubsection{Difference in confidence between data}

For this estimation, we gathered a large amount of data from a wide variety of sources, developmental stages, and environments (e.g., aquaculture vs. in situ). Even though DEB theory provides a full life cycle model with a limited number of parameters, their calibration requires a major effort. To accurately calibrate these parameters, a large diversity of datasets is necessary. Moreover, DEB theory assumes that the parameter set is individual-specific. Thus, the estimated parameter set corresponds to that of a mean individual. However, distributions of individual parameter values may depend on the population considered (i.e., different genotypes).

Consequently, the mean individual can be different from one dataset to another, whereas only one parameter set is estimated. To deal with that constraint, we made choices about model adjustment according to our objectives (e.g., favour adjustment to juvenile datasets). We also adjusted weight coefficients depending on the confidence in the quality of each dataset (e.g., uncertainty about environmental conditions or measurements) and our priorities for this application. Our choices about weight coefficients used in AmP mydata Matlab ${ }^{\circledR}$ file (see SI files) and model adjustment for the previously presented datasets are further argued below.

\section{Larvae stage}

Due to a lack of sufficient data to estimate metabolic response to temperature changes on larval stage, we assumed that temperature parameters were constant over life and wanted these estimates to account for juvenile ones. As a consequence, a poorer adjustment on zero-variate for hatching, birth, and metamorphosis stages was not considered as a sufficient argument to reject a parameter set. However, tolerance range may be narrower during early life stages (Rijnsdorp et al., 2009).

\section{Temperature tolerance}

Considering constant temperature parameters, we were not able to reproduce the temperature influence on ingestion for all fish lengths and for all temperatures from the univariate data from Fonds \& Saksena (1977). Thus, we chose to modulate these data weights to get the best fit at most common temperature encountered in the Gironde estuary $\left(10-20^{\circ} \mathrm{C}\right)$. However, a more complex response to temperature through juvenile stage and growth would probably be necessary to enhance the realism of the model, particularly in climate change-related studies. 


\section{Coherence between in situ food availability, growth and ultimate length}

Data about ultimate lengths, age-length relationships ( $t L$ and $t L \_m$ ) and length-weight relationships (LWW and LWW_m) are from the same source (Deniel, 1981) and concerns a commercially exploited fish population (Douarnenez Bay, Brittany, France). As a consequence, we chose to estimate a unique value of scaled functional response $(f)$ for all these data. To ensure that this $f$ estimate was realistic, we approximated this value over the lifespan by dividing the asymptotic length observed in the studied environment (Linf) by the theoretical maximal length that an animal can attain if the food is not limited (Lmax) (Kooijman, 2010). For males from Douarnenez Bay: Linf $=42 \mathrm{~cm}$ total length (TL) (Deniel, 1981). Lmax is estimated as the largest fish ever observed: Lmax $=75 \mathrm{~cm}$ total length (from 70 cm standard length (SL) measurement from Quéro, Desoutter, \& Lagardère (1986) and TL-SL correction factor (Froese and Pauly, 2018)). Thus, we expected $f$ estimate to be around 0.56.

However, we allowed $f$ estimate to slightly deviate from this approximation. Indeed, this approximation required ultimate lengths, whereas fish from ultimate length data were 10 to 20 years older than the major part of the population sampled. Hence, food availability may have fluctuated during a 20 years period, and the older ones may not be representative of the environmental conditions of the majority of the population. Thus, we gave less confidence to a subset of univariate data of age-length relationship ( $t L$ and $t L \_m$ ) composed of fish older than 12 years old.

As this dataset concerns a commercially exploited fish population, we were more confident about the male ultimate lengths, as they are smaller than females. However, we gave an even greater confidence to univariate relationships of growth ( $t L$ and $L W W$ ) as we wanted to catch these relationships more than the ultimate lengths, that are depending on an $f$ from possibly different earlier conditions.

\section{Puberty stage}

We chose to use actual observations of first gonadal activity as being the puberty stage for male, but not for female. Indeed, sole females need approximately 1 year to mature their oocytes (Deniel, 1981). Thus, one year was subtracted to the age at first reproduction (3.6 years old). This age was itself estimated from minimum length at first reproduction $(33 \mathrm{~cm}$, from Deniel's conclusions based on observations of gonado-somatic index and ovarian histological sections) and age-lentgh relationship for female (from Deniel's work too). Length and weight of a female of 2.6 years old was then estimated using Deniel's age-length and length-weight relationships. The poorer adjustment for female compared to male may be explained by this approximation methodology that requires multiple transformations. Indeed, we would have expected the estimated age at first reproduction (3.6 years 
old) to be closer to a round number as reproduction coincides with birth. We assume that first females that reproduce may be the bigger ones. As a consequence, we would have overestimated their age using a von Bertalanffy equation for a mean individual.

\section{Female egg production}

Female egg production depends on the amount of energy stored in the reproduction buffer (which depends on DEB parameters) and on the energy content of one egg.

We are quite confident about the zero-variate data of energy content of one egg as its estimation thanks to two references and two methods gave the same result. One method consisted in converting total lipids and crude proteins contents measured in 100\% viable batches from wild fish (Devauchelle et al., 1987) into energy content thanks to estimates of biomolecule energy densities (37500 J/g dw for total lipids and $18000 \mathrm{~J} / \mathrm{g}$ dw for crude proteins). The second method used by Flüchter \& Pandian (1968) consisted in calorific measurements of 14 groups of 300 eggs each. Results were respectively $2.02 \mathrm{~J}$ and $2.03 \mathrm{~J}$ for each method.

We are also quite confident about annual egg production data. Indeed, length-annual egg production data (LN) was measured by counting maturing oocytes $(>240 \mu \mathrm{m})$ in female gonads just before reproduction that presented no histological sign of spawning. This histological method for fecundity estimation is relevant for common sole as its reproduction is deterministic (i.e. annual egg production is fixed each year at the beginning of the gonadal maturation) and atresia is infrequent (Deniel, 1981). As a consequence, all maturing eggs observed will be spawned during the following year.

However, the actual DEBtool function used to predict annual reproduction from fish length computes it by multiplying daily egg production of a fish of the given length by 365 days. This function was developed to compute annual reproduction at ultimate length (i.e. with no growth). In our dataset, as females are still growing, their length at sampling time (i.e. used in LN dataset) is bigger than their length during the previous 365 days. Consequently, the annual production computed is overestimated by using the daily egg production at the length just before spawning period for the smallest females that are still growing (Figure S1). For this reason, we gave less confidence to data from females less than $40 \mathrm{~cm}$. Model fit to these same observations from Douarnenez bay, but considering the reproduction rules from the present-AmP model, was computed with our $\mathrm{R}$ implemented DEB model (Figure S2). Indeed, it confirmed that when the accumulation of energy in the reproduction buffer is followed through time during the simulation, predicted fecundity for the smallest females are lower. 


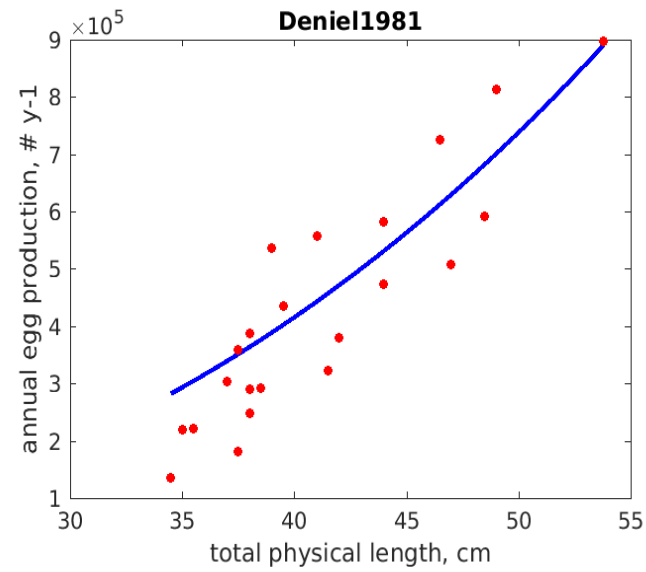

Figure S1 - Annual egg predictions from DEBtool estimation from daily egg production with a constant length all over the year.

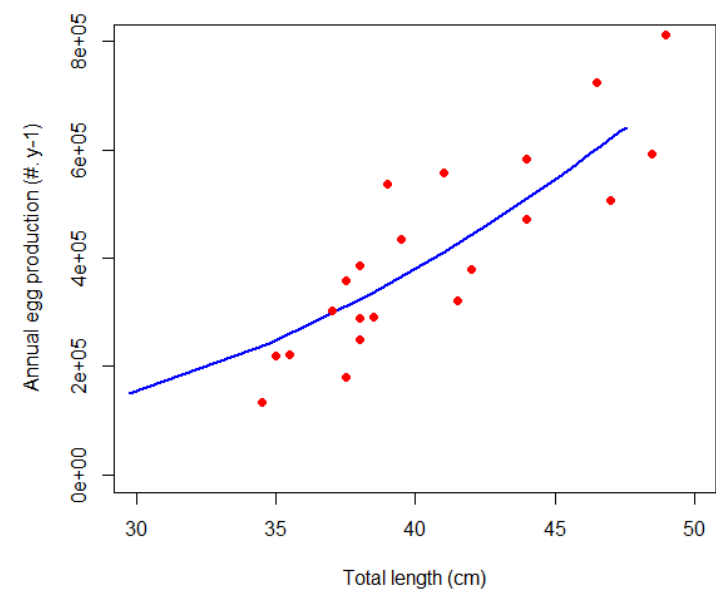

Figure S2 - Annual egg predictions from gametes buffer at length divided by the energy content of one egg.

\subsection{Male estimation of $\kappa_{R}$}

After the estimation of the other DEB parameters, we calibrated $\kappa_{\mathrm{R}}$ for males based on the difference of weight loss during reproduction period between females and males. Indeed, Rijnsdorp \& Ibelings (1989) estimated that, for North Sea plaice of $35 \mathrm{~cm}$ body length, the ratio of male over female weight losses during the spawning period was about $47 \%$. We assumed that this ratio was similar for sole.

For the DEB model simulation for soles in Douarnenez bay $\left(f=0.59758, T=14^{\circ} \mathrm{C}\right)$, we computed the loss of weight between 31th of December to $1^{\text {st }}$ of April for male and female at 10 years. This period starts before the first male batch and after the last male batch to cover the actual reproduction period for common sole used in the model. We used the value at 10 years to have little impact of growth on weight evolution.

We estimated the value of $\kappa_{R}$ necessary to predict a ratio of male over female weight losses during reproduction period that is closest to $47 \%$.

\subsection{Temperature correction factor}

In this model, temperature correction factor $(c T)$ depends on 5 parameters. We used values from Teal et al. (2012) for rates of decrease at boundaries $\left(T_{A L}\right.$ and $\left.T_{A H}\right)$ as it was the only one available for common sole. We chose to use boundary values $\left(T_{L}\right.$ and $\left.T_{H}\right)$ from Freitas et al. (2010) to have a greater correction factor between 7 and $10^{\circ} \mathrm{C}$. Indeed, as it is the natural range of temperature from December to March in the Gironde estuary (SW France, see the companion paper) we did not want $c T$ 
to be close to zero, which is observed with Teal's values. The remaining parameter describing the slope of correction factor within the tolerance range, the Arrhenius temperature $\left(T_{A}\right)$, was then estimated with DEBtool package in the same way as other DEB parameters. Parameter values are presented for the different models are reported in Table S5 and resulting temperature correction factor ( $c T$ ) evolutions with temperature are represented in Figure S3. Freitas et al. (2010) and Teal et

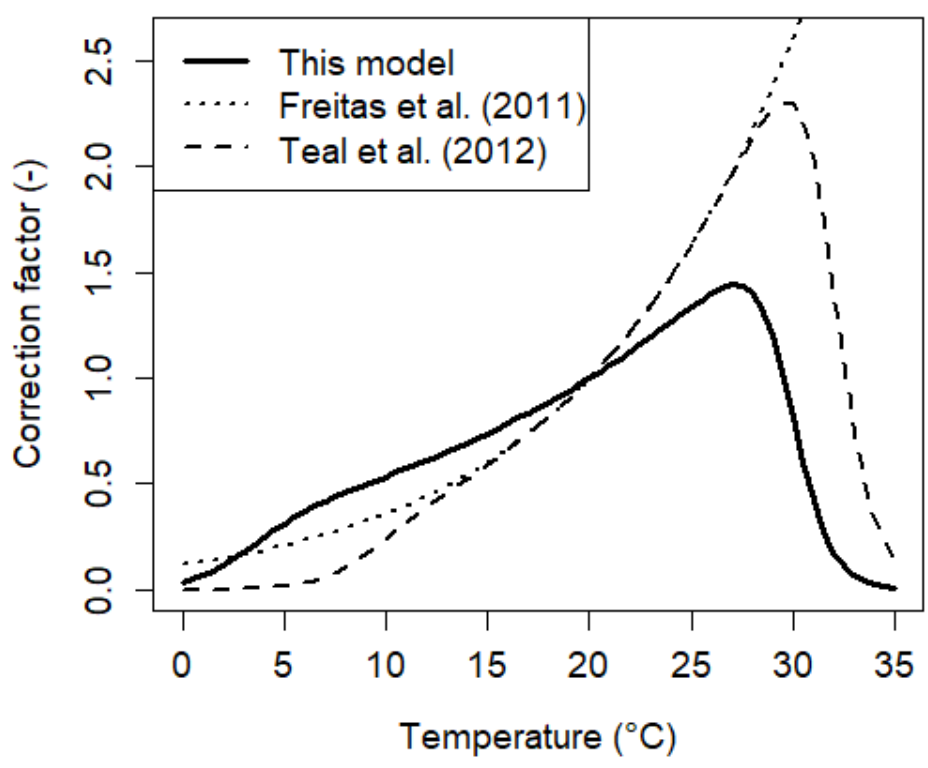

al. (2012)

Figure S3 - Temperature evolution of temperature correction factor from our model and the two references used.

Table S5 - Temperature correction parameters estimates from our model and the two references used.

\begin{tabular}{l|ccccc} 
& $\boldsymbol{T}_{\boldsymbol{A}}$ & $\boldsymbol{T}_{\boldsymbol{L}}$ & $\boldsymbol{T}_{\boldsymbol{H}}$ & $\boldsymbol{T}_{\boldsymbol{A} \boldsymbol{}}$ & $\boldsymbol{T}_{\boldsymbol{A H}}$ \\
\hline Mounier & $\mathbf{5 1 2 0}$ & $\mathbf{2 7 6}$ & $\mathbf{3 0 3}$ & $\mathbf{5 0 0 0 0}$ & $\mathbf{1 0 0 0 0 0}$ \\
Freitas & 8500 & 276 & 303 & - & - \\
Teal & 8500 & 282 & 305 & 50000 & 100000
\end{tabular}




\section{Material and methods and results for experimental datasets on larval stage}

All experiments were conducted in strict compliance with the Guide for the Care and Use of Laboratory Animals.

\subsection{Experimental set-up to house common sole larvae in 24 -well plates}

Chronology of the experimental conditions for rearing larvae is summarised in Table S6.

Solea solea eggs were obtained from a broodstock in the Netherlands (Zeeschelp, Kamperland). Sole adults naturally spawned in several batches from the $22^{\text {nd }}$ to the $24^{\text {th }}$ of April at the facilities in the Netherlands. Eggs arrived at the Ifremer facilities in La Rochelle France on the $25^{\text {th }}$ of April. Once in the lab, fertilized eggs were sorted under the stereomicroscope (Olympus, SZX9) and transferred to glass

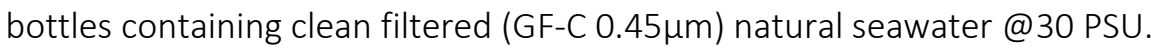

Bottles with fertilized eggs were placed in an environmental chamber (Sanyo MLR-351) with constant temperature $\left(16 \pm 1^{\circ} \mathrm{C}\right)$, oxygen, and photoperiod conditions (9h light, 15h darkness). For separating fertilized and no fertilized eggs, observation under a stereomicroscope was employed. The eggs were monitored daily and hatched during the weekend of $27^{\text {th }}$ and $28^{\text {th }}$ April 2019 (between 4 and 5 days post-fertilization depending on the batch).

Two days post-hatching (dph), 72 larvae were transferred to three 24-well polyethylene microplates. The plates were first prepared by adding $1950 \mu$ l of filtered seawater (30 ppm) per well, and then by incorporating the larvae. Translocation of the newly hatched larvae was performed one by one using a $200 \mu /$ capacity micropipette with a tip cut to avoid damage to the entering larvae. Larvae were gently pipetted on a volume of $50 \mu \mathrm{l}$ and transferred to the wells. Microplates were placed within an environmental chamber above a dark surface and incubated on the same conditions described above. Survival, malformations, behaviour, and metamorphic stages, were monitored daily for 33 days using an Olympus SZX9 stereoscope. Water was changed every other day, by carefully removing as much as possible of the water $(\sim 1950 \mu /)$ while moving the tip away from the larvae to avoid suctioning it. 
Table S6 - Chronology of the experimental conditions for rearing larvae in 24-well microplates.

\begin{tabular}{|c|c|c|c|c|c|c|c|}
\hline & Date & dpf $\mathrm{c}$ & \begin{tabular}{|l|l}
$\mathrm{dph}$ & $\mathrm{s}$ \\
$\mathrm{r}$
\end{tabular} & \begin{tabular}{|l|}
$\begin{array}{l}\text { Size } \\
\text { measured }\end{array}$ \\
\end{tabular} & \begin{tabular}{|l|}
$\begin{array}{l}\text { Water } \\
\text { changed }\end{array}$ \\
\end{tabular} & \begin{tabular}{|l|}
$\begin{array}{l}\text { Artemia/ } \\
\text { well }\end{array}$ \\
\end{tabular} & Comments \\
\hline$T$ & $23 / 04 / 2019$ & 0 & & & & & Spawning at Zeeschelp, Kamperland the Netherlands \\
\hline $\mathrm{W}$ & $24 / 04 / 2019$ & 1 & & & & & Spawning at Zeeschelp, Kamperland the Netherlands \\
\hline$T$ & $25 / 04 / 2019$ & 2 & & & & & Arrival to Ifremer facilities, La Rochelle France \\
\hline $\mathrm{F}$ & $26 / 04 / 2019$ & 3 & & & & & \\
\hline S & 27/04/2019 & 4 & 1 & & & & Hatching \\
\hline$S$ & $28 / 04 / 2019$ & 5 & 2 & & & & \\
\hline $\mathrm{M}$ & $\mid 29 / 04 / 2019$ & 6 & 3 & & $\mathrm{x}$ & & Transfer to microplates \\
\hline $\mathbf{T}$ & 30/04/2019 & 7 & 4 & & & & \\
\hline w & 01/05/2019 & 8 & 5 & & $\mathrm{x}$ & 5 & Start feeding \\
\hline $\mathbf{T}$ & $02 / 05 / 2019$ & 9 & 6 & & & 5 & \\
\hline $\mathrm{F}$ & 03/05/2019 & 10 & 7 & $\mathrm{x}$ & $\mathrm{x}$ & 7 & \\
\hline S & 04/05/2019 & 11 & 8 & & & 14 & Artemia added for Saturday and Sunday \\
\hline S & 05/05/2019 & 12 & 9 & & & & not fed \\
\hline $\mathrm{M}$ & 06/05/2019 & 13 & 10 & & $\mathrm{x}$ & 15 & \\
\hline$T$ & 07/05/2019 & 14 & 11 & & & 20 & \\
\hline W & 08/05/2019 & 15 & 12 & & $\mathrm{x}$ & 20 & \\
\hline$T$ & |09/05/2019 & 16 & 13 & & & 20 & \\
\hline $\mathrm{F}$ & $10 / 05 / 2019$ & 17 & 14 & $x$ & $\mathrm{x}$ & 20 & \\
\hline S & $11 / 05 / 2019$ & 18 & 15 & & & 20 & \\
\hline S & $12 / 05 / 2019$ & 19 & 16 & & & 20 & \\
\hline $\mathrm{M}$ & $\mid 13 / 05 / 2019$ & 20 & 17 & & $\mathrm{x}$ & 20 & \\
\hline$T$ & $14 / 05 / 2019$ & 21 & 18 & & & 25 & \\
\hline $\mathrm{w}$ & $15 / 05 / 2019$ & 22 & 19 & & $\mathrm{x}$ & 25 & \\
\hline $\mathbf{T}$ & $16 / 05 / 2019$ & $23 \mid$ & 20 & & & 30 & Start of metamorphosis \\
\hline $\mathrm{F}$ & $\mid 17 / 05 / 2019$ & 24 & 21 & $\mathrm{x}$ & $\mathrm{x}$ & 30 & \\
\hline$S$ & $18 / 05 / 2019$ & 25 & 22 & & & 70 & Artemia added for Saturday and Sunday \\
\hline $\mathrm{S}$ & $19 / 05 / 2019$ & 26 & 23 & & & & not fed \\
\hline $\mathrm{M}$ & 20/05/2019 & 27 & 24 & & $\mathrm{x}$ & 40 & \\
\hline$T$ & $\mid 21 / 05 / 2019$ & 28 & 25 & & & 40 & \\
\hline $\mathrm{w}$ & $22 / 05 / 2019$ & 29 & 26 & & $\mathrm{x}$ & 40 & \\
\hline$T$ & $23 / 05 / 2019$ & 30 & 27 & & & 40 & \\
\hline $\mathrm{F}$ & 24/05/2019 & 31 & 28 & & $\mathrm{x}$ & 50 & \\
\hline$S$ & $25 / 05 / 2019$ & 32 & 29 & & & 80 & Artemia added for Saturday and Sunday \\
\hline$S$ & $26 / 05 / 2019$ & 33 & 30 & & & & not fed \\
\hline $\mathrm{M}$ & $27 / 05 / 2019$ & 34 & 31 & & $x$ & 50 & \\
\hline $\mathbf{T}$ & 28/05/2019 & 35 & 32 & & & 50 & \\
\hline $\mathrm{W}$ & $29 / 05 / 2019$ & 36 & 33 & & & & End of the experiment \\
\hline
\end{tabular}

\subsection{Feeding, metamorphosis and growth monitoring}

From day 5 post-hatching onwards, larvae were fed with Artemia salina (Sep-Art Artemia Cyst, Ocean Nutrition, easily separable brine shrimp eggs prepared according to manufacturer instructions). We followed the number of artemias ate per larvae, by monitoring both, the quantity of food provided and consumed per larvae each day. On the first day, larvae were fed five artemias, an amount that increased following the percentage of food consumed daily. The objective was to provide food ad libitum without compromising the quality of the water, and the experimental conditions for the sole. Please refer to Table S7 to know the exact amount of artemia we fed the larvae until the end of the experiment. 
Metamorphic stage was estimated at the end of the experiment. A score of metamorphosis completion ranging from 0 to 9 was calculated by counting the number of fulfilled criteria from the following list:

- left eye separates from the eye socket;

- left eye is close to the mid-line of the dorsal surface;

- larva is asymmetrical;

- left eye migration exceeded the dorsal mid-line and can be observed from the top side;

- left eye movement is finished,

- head is completely oval and remodelled;

- dorsal mid-line is completely oval and remodelled;

- digestive system is relocated;

- digestive system is oval.

\subsection{Length and weight measurements}

The length of a total of 12 larvae was monitored every week, starting at $7 \mathrm{dph}$ until the end of the experiment at $33 \mathrm{dph}$. For that, we translocated the larvae to a microscope calibration slide (grid 1 $\mathrm{mm}$ ) in a small volume (circa $50 \mu \mathrm{l})$. Larvae length was recorded using the same stereoscope connected to a camera (The Imaging Source, DFK 31AU03). For all length pictures, colour lighting was set to $2850 \mathrm{~K}$ (Schott KL 1500 LCD cold light source), and the stereoscope magnification was between 10-12.5, which corresponds to $120-123 \mathrm{pixel} / \mathrm{mm}$. All length measurements were performed with the free Java image processing program ImageJ (Abràmoff et al., 2004). At the end of the experiment, all larvae were collected and wet and dry weight measured. For dry weight calculations, larvae were placed at the stove for $24 \mathrm{~h}$ at $60^{\circ} \mathrm{C}$ using a Mettler analytical Balance model AE240. 


\subsection{Raw datasets}

Dataset of individual daily food ingestion used for simulation purpose are presented in Table S7. The individual "3_D1" was not considered in the analyses as it died before the end of the experiment. Table S8 presents the percent of distributed food that was actually eaten by larva. Table S9 contains growth monitoring and Table S10 final weights.

Table S7 - Individual daily food ingestion of larvae in number of artemias adapted for simulation purpose (highlighted columns correspond to the estimations for non feeding days).

\begin{tabular}{|c|c|c|c|c|c|c|c|c|c|c|c|c|c|c|c|c|c|c|c|c|c|c|c|c|c|c|c|c|c|c|}
\hline TAG | Age (dph) & 4 & 5 & 6 & 7 & 8 & 9 & 10 & 11 & 12 & 13 & 14 & 15 & 16 & 17 & 18 & 9 & 20 & 21 & 22 & 23 & 24 & 25 & 26 & 27 & 28 & 29 & 30 & 31 & 32 & 33 \\
\hline 1_A1 & 0 & 4 & 5 & 6 & 9 & 9 & 14 & 10 & 18 & 19 & 18 & 19 & 19 & 18 & 25 & 25 & 29 & 30 & 35 & 35 & 40 & 40 & 40 & 40 & 50 & 39 & 39 & 49 & 50 & 0 \\
\hline 1_B1 & 0 & 2 & 8 & 7 & 7 & 7 & 13 & 18 & 20 & 19 & 16 & 16 & 19 & 20 & 24 & 25 & 26 & 26 & 35.5 & 35.5 & 39 & 35 & 39 & 39 & 48 & 41 & 41 & 49 & 50 & 0 \\
\hline 1_C1 & 0 & 4 & 5 & 6 & 9.5 & 9.5 & 15 & 16 & 19 & 19 & 18 & 20 & 19 & 19 & 21 & 25 & 32 & 31 & 35 & 35 & 40 & 40 & 40 & 37 & 50 & 40 & 40 & 50 & 50 & 0 \\
\hline 1_D1 & 0 & 1 & 8 & 3 & 8 & 8 & 15 & 16 & 20 & 19 & 21 & 19 & 18 & 20 & 24 & 25 & 30 & 30 & 35 & 35 & 40 & 40 & 38 & 40 & 50 & 39 & 39 & 50 & 48 & 0 \\
\hline 2_A1 & 0 & 0 & 5 & 4 & 6 & 6 & 4 & 12 & 15 & 15 & 18 & 20 & 20 & 20 & 25 & 25 & 28 & 23 & 37 & 37 & 38 & 40 & 38 & 37 & 50 & 39.5 & 39.5 & 50 & 50 & 0 \\
\hline 2_B1 & 0 & 5 & 5 & 7 & 10 & 10 & 13 & 16 & 19 & 17 & 19 & 19 & 17 & 19 & 23 & 25 & 30 & 30 & 35 & 35 & 39 & 40 & 40 & 40 & 50 & 40 & 40 & 50 & 50 & 0 \\
\hline 2_C1 & 0 & 5 & 5 & 7 & 7.5 & 7.5 & 20 & 19 & 20 & 18 & 21 & 20 & 20 & 20 & 24 & 24 & 25 & 30 & 35 & 35 & 40 & 39 & 40 & 40 & 50 & 40 & 40 & 50 & 48 & 0 \\
\hline 2_D1 & 0 & 5 & 5 & 7 & 7.5 & 7.5 & 15 & 16 & 20 & 20 & 17 & 17 & 19 & 20 & 25 & 23 & 28 & 30 & 35 & 35 & 40 & 40 & 40 & 40 & 50 & 40 & 40 & 50 & 48 & 0 \\
\hline 3_A1 & 0 & 5 & 5 & 7 & 10 & 10 & 15 & 15 & 12 & 9 & 5 & 18 & 16 & 19 & 21 & 21 & 21 & 29 & 35 & 35 & 40 & 40 & 39 & 39 & 48 & 41 & 41 & 50 & 50 & 0 \\
\hline 3_B1 & 0 & 5 & 5 & 6 & 9.5 & 9.5 & 15 & 18 & 20 & 15 & 20 & 21 & 21 & 18 & 21 & 24 & 30 & 30 & 35 & 35 & 40 & 34 & 40 & 33 & 44 & 40 & 40 & 48 & 34 & 0 \\
\hline 3_C1 & 0 & 5 & 5 & 7 & 10 & 10 & 15 & 20 & 20 & 15 & 19 & 20 & 20 & 19 & 24 & 25 & 30 & 30 & 35 & 35 & 40 & 40 & 40 & 40 & 50 & 40 & 40 & 50 & 50 & 0 \\
\hline 3_D1 & 0 & 5 & 5 & 6 & 10 & 10 & 14 & 20 & 18 & 20 & 14 & 20 & 20 & 13 & 16 & 22 & 28 & 29 & 35.5 & 35.5 & 40 & NA & NA & NA & NA & NA & NA & NA & NA & NA \\
\hline
\end{tabular}


Table S8 - Individual daily proportion of food distributed having been ingested for each larva in percent (highlighted columns correspond to the estimations for non feeding days).

\begin{tabular}{|c|c|c|c|c|c|c|c|c|c|c|c|c|c|c|c|c|c|c|c|c|c|c|c|c|c|c|c|}
\hline TAG | Age (dph) & 4 & 5 & 6 & 7 & 8 & 10 & 11 & 12 & 13 & 14 & 15 & 16 & 17 & 18 & 19 & 20 & 21 & $22 \quad 23$ & 24 & 25 & 26 & 27 & 28 & 2930 & 31 & 32 & 33 \\
\hline 1_A1 & NA & 100 & 100 & 75 & 95 & 93 & 50 & 90 & 95 & 90 & 95 & 95 & 90 & 100 & 100 & 97 & 100 & 100 & 100 & 100 & 100 & 100 & 100 & 98 & 98 & 100 & NA \\
\hline 1_B1 & NA & 40 & 100 & 100 & 70 & 87 & 90 & 100 & 95 & 80 & 80 & 95 & 100 & 96 & 100 & 87 & 87 & 96 & 98 & 88 & 98 & 98 & 96 & 100 & 98 & 100 & NA \\
\hline 1_C1 & NA & 100 & 100 & 86 & 95 & 100 & 80 & 95 & 95 & 90 & 100 & 95 & 95 & 84 & 100 & 100 & 97 & 99 & 100 & 100 & 100 & 93 & 100 & 100 & 100 & 100 & NA \\
\hline 1_D1 & NA & 20 & 89 & 43 & 80 & 100 & 80 & 100 & 95 & 100 & 95 & 90 & 100 & 96 & 100 & 100 & 100 & 100 & 100 & 100 & 95 & 100 & 100 & 98 & 100 & 96 & NA \\
\hline 2_A1 & NA & 0 & 50 & 57 & 57 & 27 & 60 & 75 & 75 & 90 & 100 & 100 & 100 & 100 & 100 & 93 & 77 & 96 & 95 & 100 & 95 & 93 & 100 & 99 & 100 & 100 & NA \\
\hline 2_B1 & NA & 100 & 100 & 100 & 100 & 87 & 80 & 95 & 85 & 86 & 95 & 85 & 95 & 92 & 100 & 100 & 100 & 100 & 98 & 100 & 100 & 100 & 100 & 100 & 100 & 100 & NA \\
\hline 2_C1 & NA & 100 & 100 & 100 & 19 & 14 & 95 & 100 & 90 & 100 & 100 & 100 & 100 & 96 & 96 & 83 & 100 & 100 & 100 & 98 & 100 & 100 & 100 & 100 & 100 & 96 & NA \\
\hline 2_D1 & NA & 100 & 100 & 100 & 20 & 100 & 80 & 100 & 100 & 85 & 85 & 95 & 100 & 100 & 92 & 93 & 100 & 100 & 100 & 100 & 100 & 100 & 100 & 100 & 100 & 96 & NA \\
\hline 3_A1 & NA & 100 & 100 & 100 & 100 & 100 & 75 & 60 & 45 & 25 & 90 & 80 & 95 & 84 & 84 & 70 & 97 & 100 & 100 & 100 & 98 & 98 & 96 & 100 & 100 & 100 & NA \\
\hline 3_B1 & NA & 100 & 100 & 86 & 95 & 100 & 90 & 100 & 75 & 100 & 100 & 100 & 86 & 84 & 96 & 100 & 100 & 100 & 100 & 85 & 100 & 83 & 88 & 93 & 96 & 68 & NA \\
\hline 3_C1 & NA & 100 & 100 & 100 & 100 & 100 & 100 & 100 & 75 & 100 & 100 & 100 & 95 & 96 & 100 & 100 & 100 & 100 & 100 & 100 & 100 & 100 & 100 & 100 & 100 & 100 & NA \\
\hline 3_D1 & NA & 100 & 100 & 100 & 100 & 93 & 100 & 90 & 100 & 70 & 100 & 100 & 65 & 64 & 88 & 93 & 97 & 100 & 100 & NA & NA & NA & NA & NA & NA & NA & NA \\
\hline
\end{tabular}


Table S9 - Growth monitoring of larvae (mm).

\begin{tabular}{c|ccccc} 
TAG | Age (dph) & 7 & 14 & 21 & 28 & 33 \\
\hline 1_A1 & 4.378 & 5.492 & 6.205 & 6.721 & 7.896 \\
1_B1 & 4.107 & 5.244 & 6.2 & 7.122 & 8.155 \\
1_C1 & 4.396 & 5.649 & 6.09 & 7.163 & 8.079 \\
1_D1 & 3.914 & 5.3 & 6.022 & 6.934 & 8.044 \\
2_A1 & 4.102 & 5.204 & 6.197 & 6.729 & 7.747 \\
2_B1 & 4.755 & 5.757 & 6.536 & 7.145 & 7.91 \\
2_C1 & 4.568 & 5.432 & 6.479 & 7.182 & 8.395 \\
2_D1 & 4.33 & 4.894 & 6.136 & 7.139 & 8.126 \\
3_A1 & 4.385 & 5.458 & 6.293 & 7.139 & 8.087 \\
3_B1 & 4.195 & 4.684 & 6.407 & 7.129 & 8.162 \\
3_C1 & 4.33 & 5.324 & 6.07 & 6.354 & 7.721 \\
3_D1 & 4.347 & 5.723 & 6.111 & 6.892 & NA
\end{tabular}

Table S10 - Final dry weight of larvae (mg)

\begin{tabular}{c|c} 
TAG | Age (dph) & 33 \\
\hline 1_A1 & 0.91 \\
1_B1 & 0.93 \\
1_C1 & 1.14 \\
1_D1 & 1.02 \\
2_A1 & 0.00075 \\
2_B1 & 0.00077 \\
2_C1 & 0.00085 \\
2_D1 & 0.00085 \\
3_A1 & 0.00077 \\
3_B1 & 0.00068 \\
3_C1 & 0.00076 \\
3_D1 & NA
\end{tabular}




\section{Summary of material and methods from Eichinger et al. (2010)}

In this contamination-decontamination experiment, juvenile soles (GO) were fed daily using automated belt feeders located in one part of the tanks. The energy density of the commerciallyformulated food pellets was $21,612{\mathrm{~J} \cdot \mathrm{g}_{\text {food }}}^{-1}$. Daily rations were adjusted to the estimated growing biomass in tanks following the supplier's recommendations (assumed to be ad libitum). The water temperature was kept constant at $19^{\circ} \mathrm{C}$. Fish were divided into two groups: PCB and control. No difference in growth was observed between the juveniles from the two groups during this experiment. Only fish from the PCB group are used in this paper. These ones were given food pellets with a mixture of 4 PCBs (i.e. CB105, CB118, CB149 and CB153, see Eichinger et al., 2010 for PCB quantification procedure). During the contamination period, fish were exposed to contaminated food during 84 days

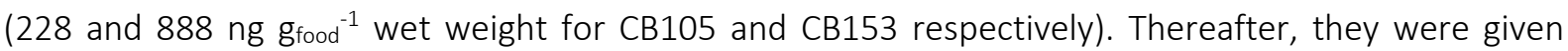
food pellets without PCBs $\left(<3 \mathrm{ng} \mathrm{g}{ }^{-1}\right.$ ww for all CBs studied, see Eichinger et al., 2010 for PCB quantification procedure) during a decontamination period of 84 days. 


\section{Literature cited}

Abràmoff, M.D., Magalhães, P.J., Ram, S.J., 2004. Image processing with imageJ. Biophotonics Int. 11, 36-41. https://doi.org/10.1201/9781420005615.ax4

Day, O.J., Jones, D.A., Howell, B.R., 1996. Food consumption, growth and respiration of sole, Solea solea (L.), during early ontogeny in a hatchery environment. Aquac. Res. 27, 831-839. https://doi.org/10.1046/j.1365-2109.1996.t01-1-00800.x

Deniel, C., 1981. Les poissons plats [Téléostéens, Plueornectiformes] en baie de Douarnenez: reproduction, croissance et migration. J. Fish Biol. Université de Bretagne Occidentale - Brest.

Devauchelle, N., Alexandre, J.C., Corre, N.L.E., Letty, Y., 1987. Spawning of Sole ( Solea solea ) in Captivity. Aquaculture 66, 125-147.

Dorel, D., 1986. Poissons de l'Atlantique Nord-Est, Relations Taille-Poids. Nantes.

Flüchter, J., Pandian, T.J., 1968. Rate and efficiency of yolk utilization in developing eggs of the sole Solea solea. Helgol??nder Wissenschaftliche Meeresuntersuchungen 18, 53-60. https://doi.org/10.1007/BF01611665

Fonds, M., 1979. Laboratory Observations on the Influence of Temperature and Salinity on Development of the Eggs and Growth of the Larvae of Solea solea (Pisces). Mar. Ecol. Prog. Ser. 1, 91-99. https://doi.org/10.3354/meps001091

Fonds, M., Drinkwaard, B., Resink, J.W., Eysink, G.G.J., Toet, W., 1989. Measurements of metabolism, food intake and growth of Solea solea (L.) fed with mussel meat or with dry food, in: Aquaculture: A Biotechnology in Progress: Volume 2. pp. 851-874.

Fonds, M., Saksena, V.P., 1977. The daily food intake of young soles (Solea solea L,) in relation to their size and the water temperature. I.C.E.S. Work. Gr. Maric. 4, 51-58.

Freitas, V., Cardoso, J.F.M.F., Lika, K., Peck, M.A., Campos, J., Kooijman, S.A.L.M., van der Veer, H.W., 2010. Temperature tolerance and energetics: a dynamic energy budget-based comparison of North Atlantic marine species. Philos. Trans. R. Soc. B Biol. Sci. 365, 3553-3565. https://doi.org/10.1098/rstb.2010.0049

Froese, R., Pauly, D., 2018. FishBase. World Wide Web Electron. Publ.

Galois, R., Lagardere, F., Richard, P., 1990. Changes in biochemical composition and otolith microstructure of larval common soleas, Solea solea (L.) under experimental starvation. La Mer.

Kooijman, S.A.L.M., 2010. Dynamic Energy Budget Theory for Metabolic Organisation - Third edition. https://doi.org/10.3867/j.issn.1000-3002.2017.04.008

Lika, K., Kearney, M.R., Freitas, V., van der Veer, H.W., van der Meer, J., Wijsman, J.W.M., Pecquerie, L., Kooijman, S.A.L.M., 2011. The "covariation method" for estimating the parameters of the standard Dynamic Energy Budget model I: Philosophy and approach. J. Sea Res. https://doi.org/10.1016/j.seares.2011.07.010

Lika, K., Kooijman, S.A.L.M., Papandroulakis, N., 2014. Metabolic acceleration in Mediterranean Perciformes. J. Sea Res. 94, 37-46. https://doi.org/10.1016/j.seares.2013.12.012

Lund, I., Steenfeldt, S.J., Suhr, K.I., Hansen, B.W., 2008. A comparison of fatty acid composition and quality aspects of eggs and larvae from cultured and wild broodstock of common sole ( Solea 
solea L . ). Aquac. Nutr. 14, 544-555. https://doi.org/10.1111/j.1365-2095.2007.00560.x

Quéro, J.-C., Desoutter, M., Lagardère, F., 1986. Soleidae, in: Fishes of the North-Eastern Atlantic and the Mediterranean, Volume 3. pp. 1308-1324.

Rijnsdorp, A.D., Ibelings, B., 1989. Sexual dimorphism in the energetics of reproduction and growth of North Sea plaice, Pleuronectes platessa L. J. Fish Biol. 35, 401-415. https://doi.org/10.1111/j.1095-8649.1989.tb02992.x

Rijnsdorp, A.D., Peck, M.A., Engelhard, G.H., Mollmann, C., Pinnegar, J.K., 2009. Resolving the effect of climate change on fish populations. ICES J. Text 1570-1583.

Teal, L.R., van Hal, R., van Kooten, T., Ruardij, P., Rijnsdorp, A.D., 2012. Bio-energetics underpins the spatial response of North Sea plaice (Pleuronectes platessa L.) and sole (Solea solea L.) to climate change. Glob. Chang. Biol. 18, 3291-3305. https://doi.org/10.1111/j.13652486.2012.02795.x

van der Veer, H.W., Kooijman, S.A.L.M., van der Meer, J., 2001. Intra- and interspecies comparison of energy flow in North Atlantic flatfish species by means of dynamic energy budgets. J. Sea Res. 45, 303-320. https://doi.org/10.1016/S1385-1101(01)00061-2

Wiki style AmP manual [WWW Document], n.d. URL http://www.debtheory.org/wiki/index.php?title=Add-my-pet_Introduction (accessed 1.22.18). 